\title{
The Ital-FLAMES survey of the Sagittarius dwarf spheroidal galaxy
}

\section{Chemical abundances of bright RGB stars ${ }^{\star} \star \star$}

\author{
L. Monaco ${ }^{1,2}$, M. Bellazzini' ${ }^{2}$, P. Bonifacio ${ }^{1}$, F. R. Ferraro $^{3}$, \\ G. Marconi ${ }^{4}$, E. Pancino ${ }^{2}$, L. Sbordone ${ }^{5,6}$, and S. Zaggia ${ }^{1}$ \\ 1 Istituto Nazionale di Astrofisica - Osservatorio Astronomico di Trieste, via Tiepolo 11, 34131 Trieste, Italy \\ e-mail: 1monaco@eso.org \\ 2 Istituto Nazionale di Astrofisica - Osservatorio Astronomico di Bologna, 40127 Bologna, Italy \\ 3 Università di Bologna - Dipartimento di Astronomia, 40127 Bologna, Italy \\ ${ }^{4}$ European Southern Observatory, Casilla 19001, Santiago, Chile \\ 5 Istituto Nazionale di Astrofisica - Osservatorio Astronomico di Roma, via Frascati 33, 00040 Monteporzio Catone, \\ Roma, Italy \\ ${ }^{6}$ Università Tor Vergata, Roma, Italy
}

Received 28 April 2005 / Accepted 22 June 2005

\begin{abstract}
We present iron and $\alpha$ element $(\mathrm{Mg}, \mathrm{Ca}, \mathrm{Ti})$ abundances for a sample of 15 Red Giant Branch stars belonging to the main body of the Sagittarius dwarf Spheroidal galaxy. Abundances have been obtained from spectra collected using the high resolution spectrograph FLAMES-UVES mounted at the VLT. Stars of our sample have a mean metallicity of $[\mathrm{Fe} / \mathrm{H}]=$ $-0.41 \pm 0.20$ with a metal-poor tail extending to $[\mathrm{Fe} / \mathrm{H}]=-1.52$. The $\alpha$ element abundance ratios are slightly subsolar for metallicities higher than $[\mathrm{Fe} / \mathrm{H}] \gtrsim-1$, suggesting a slow star formation rate. The $[\alpha / \mathrm{Fe}]$ of stars having $[\mathrm{Fe} / \mathrm{H}]<-1$ are compatible to what observed in Milky Way stars of comparable metallicity.
\end{abstract}

Key words. stars: atmospheres - Galaxy: abundances - galaxies: evolution - galaxies: dwarf - galaxies: individual: Sgr dSph

\section{Introduction}

The Local Group (LG) is a heterogeneous environment. Galaxies in the LG show a variety of characteristics (e.g. mass, morphology, gas content) and are evolving under different conditions (e.g. in isolation, on strong dynamical interaction). Thererore, in principle, they could teach us about galaxy evolution as much as globular clusters did concerning stellar evolution. Chemical abundances and abundance ratios are key ingredients to study the star formation histories of stellar systems. The modern generation of spectrographs mounted on 8-10 m class telescopes allows us to investigate the chemical composition and dynamics of bright stars in LG galaxies but only a handful of stars have been studied so far (Tolstoy et al. 2004, 2003; Shetrone et al. 2003; Bonifacio et al. 2000, 2004; Fulbright et al. 2004; Geisler et al. 2005; Shetrone et al. 1998, 2001).

\footnotetext{
* Based on observations obtained with FLAMES at VLT Kueyen $8.2 \mathrm{~m}$ telescope in the program 71.B-0146.

$\star \star$ Appendix A is only available in electronic form at http://www.edpsciences.org
}

The commonly accepted paradigm (White \& Rees 1978) predicts the formation of large galaxies from the hierarchical assembly of small fragments similar to the LG dwarf spheroidals (dSphs). In this framework, the comparison between the chemical composition of the Milky Way (MW) and LG dSph stars is a first local testbed for the hierarchical merging model. The chemical composition of LG stars turned out to be remarkably different from that of MW stars of comparable metallicities. In particular, LG stars show $\alpha$ element abundance ratios systematically under-abundant with respect to MW stars (see, for instance, Venn et al. 2004; Bonifacio et al. 2004). The interpretation of this empirical evidence is controversial. Is the hierarchical merging a minor process in the assembly of the MW? Or were the fragments from which the MW formed at early times different from the nowadays recognizable dSphs? The chemical difference between MW and LG stars may reflect an environmental difference between dwarfs accreted at early times (galaxies near the bottom of the pre-MW potential well dense environment) and the surviving dwarfs (galaxies far from the bottom of the pre-MW potential well - loose environment, but see Robertson et al. 2005; Bullock \& Johnston 2004). 
The Sagittarius dSph (hereafter Sgr, Ibata et al. 1994) is a LG galaxy currently experiencing strong and disruptive tidal interactions with the MW (Ibata et al. 1995, 1997; Majewski et al. 2003). Therefore, it may provide clues on the influence of dynamical interactions on the chemical evolution of dwarf galaxies.

It is well-known that the complex stellar content of Sgr (see Monaco et al. 2002; Monaco et al. 2003, 2005, and references therein) is largely dominated by a population of oldintermediate age stars ( $\sim 6 \mathrm{Gyr}$, see, e.g. Bellazzini et al. 1999; Layden \& Sarajedini 2000; Monaco et al. 2002). However, some concerns have been raised on mean metallicity estimates obtained for this population from spectroscopic and photometric works (see, e.g. Mateo et al. 1995; Bonifacio et al. 2000; Cole 2001; Monaco et al. 2002; Bonifacio et al. 2004).

The paper is devoted to the assessment of the mean chemical properties of the Sgr dominant population. We present Fe, $\mathrm{Mg}, \mathrm{Ca}$ and $\mathrm{Ti}$ abundances for a selected sample of stars belonging to this population. In a companion paper (Bonifacio et al., in preparation) we deal with the issue of the Sgr metallicity distribution.

$[\mathrm{Fe} / \mathrm{H}]$ and $[\alpha / \mathrm{Fe}]$ abundances as well as the trends in the $[\mathrm{Fe} / \mathrm{H}]$ vs. $[\alpha / \mathrm{Fe}]$ plane constrain the chemical evolution which led to the formation of the Sgr dominant population (Lanfranchi \& Matteucci 2003). Moreover, mean [Fe/H] and $[\alpha / \mathrm{Fe}]$ values are key ingredients to derive reliable age estimates from the color-magnitude diagrams.

The paper is organized as follows. In Sect. 2 we describe the target selection and the obtained data. In Sect. 3 we describe the procedures followed to fix the atmospheric parameters and the chemical analysis. In Sect. 4 we compare the results obtained with previous works and in Sect. 5 we discuss our findings.

\section{Observations}

\subsection{Target selection, data and equivalent widths}

As part of the guaranteed time awarded to the Ital-FLAMES consortium, more than 400 stars were observed in the Sgr dSph (Bonifacio et al. 2005; Zaggia et al. 2004) from May the 23th to 27th, 2003, using the FLAMES facility mounted on the VLT (Pasquini et al. 2000). Details on the observations are given in Zaggia et al. (2004). FLAMES allows us to observe 132 targets in one shot using the intermediate-low resolution spectrograph GIRAFFE, plus 8 additional targets using the red arm of the high resolution specrograph UVES. In this paper we present the results obtained from the UVES spectra.

It is important to recall that a large number of Milky Way foreground stars are present along the Sgr line of sight. In order to optimize the Sgr star detection rate, the target selection for the UVES fibres was performed using the infrared 2 MASS $^{1}$ color magnitude diagram (CMD). In fact, in the infrared plane, the upper Sgr red giant branch (RGB) stands out very clearly from the contaminating MW field (see, e.g., Cole 2001). This also allows a thorough sampling of the Sgr dominant population. In Fig. 1 we plotted the 2 MASS $\left(K ; J-K_{\mathrm{S}}\right)$ CMD for

\footnotetext{
${ }^{1}$ See http://www.ipac.caltech.edu/2mass
}

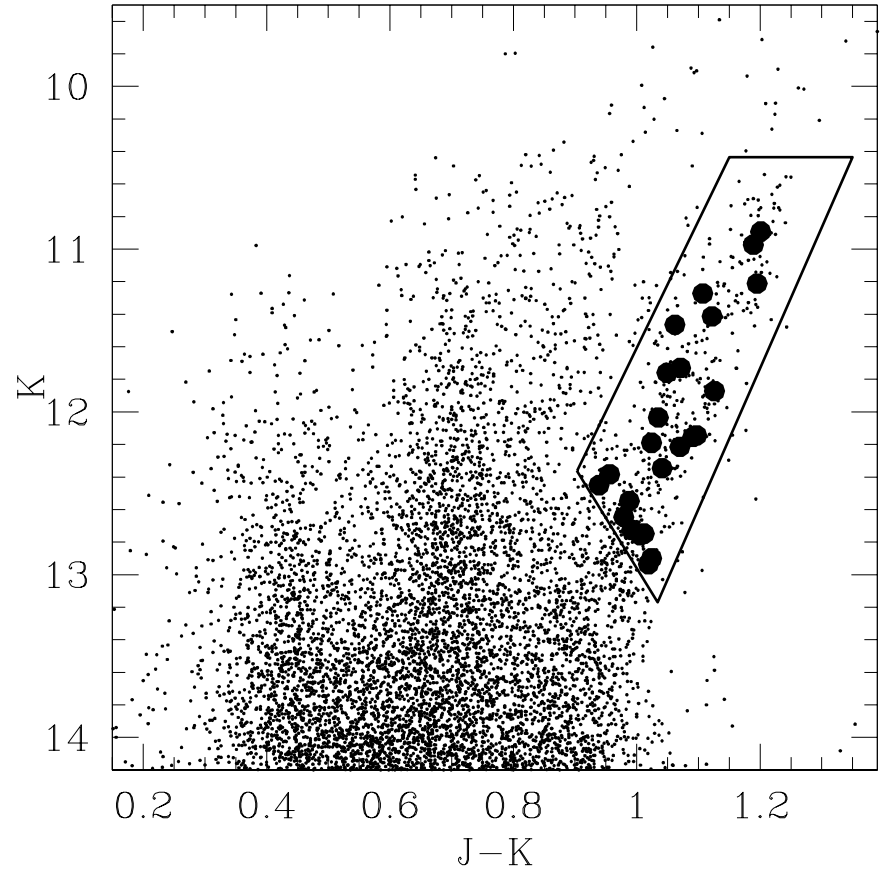

Fig. 1. The $K$ vs. $J-K_{\mathrm{S}} 2$ MASS color-magnitude diagram for a one square degree region around the globular cluster M 54. Target stars are plotted as large filled circles.

a 1 square degree area centred on the globular cluster M 54. The heavy continuous line is the selection box. Target stars are plotted as large filled circles. A similar target selection already proved to be very effective in detecting stars belonging to the Sgr Stream (Majewski et al. 2004).

Target stars are marked as large symbols in the optical CMD plotted in Fig. 2 (Monaco et al. 2002). In Table 1 we report equatorial ( $\mathrm{J} 2000.0$ ) coordinates and $V$ and $I$ magnitudes for the target stars.

The coordinates in the $\mathbf{J} 2000.0$ absolute astrometric system for both UVES and GIRAFFE samples were obtained with a procedure already described in other papers (see, for example, Ferraro et al. 2001). The new astrometric Guide Star Catalogue (GSC II) recently released and now available on the $w^{2} b^{2}$ was used as reference. In order to derive an astrometric solution we used a program specifically developed at Bologna Observatory (Montegriffo et al., in preparation). As a result of the entire procedure, rms residuals of $\sim 0.15$ arcsec, both in RA and Dec, were obtained. The quality of the astrometry was confirmed by the successful centering of the fibres.

We performed the analysis on the spectra reduced with the UVES ESO-MIDAS ${ }^{3}$ pipeline. For each pointing, 7 fibres were centred on the target stars while one fibre was used to measure the sky spectrum. Different spectra of the same star were coadded and the resulting signal to noise ratio $(S / N)$ ranges from 14 to 43 at $653 \mathrm{~nm}$ (see Table 2). UVES spectra have a

\footnotetext{
${ }^{2}$ See http://www-gsss. stsci.edu/gsc/gsc2/GSC2home.htm

3 ESO-MIDAS is the acronym for the European Southern Observatory Munich Image Data Analysis System which is developed and maintained by the European Southern Observatory. http://www.eso.org/projects/esomidas/
} 


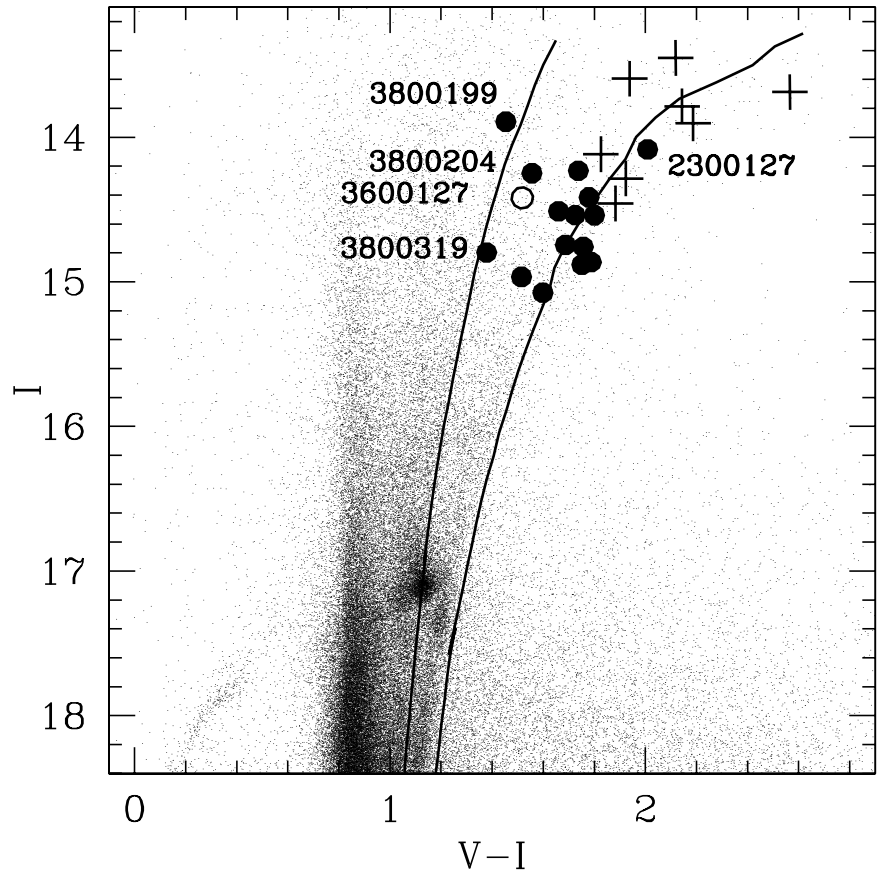

Fig. 2. $I$ vs. $V-I$ color-magnitude diagram for a one square degree region around the globular cluster M 54. Target stars are marked with large symbols. Stars showing TiO molecular bands in the spectra are plotted as plus symbols. Star 3600217 has a radial velocity not compatible with the membership to Sgr and is plotted as a large empty circle. Theoretical isochrones from which the surface gravities for the programme stars were obtained are also plotted as continuous lines.

resolution of $R \simeq 43000$ and cover the range between $480 \mathrm{~nm}$ and $680 \mathrm{~nm}$.

Equivalent widths $(E W)$ were measured on the spectra using the standard $\mathrm{IRAF}^{4}$ task splot. The $\mathrm{Fe}, \mathrm{Ca}, \mathrm{Mg}$ and Ti line lists as well as the adopted atomic parameters and the measured $E W$ are reported in Table A.1. A different iron line list (see Table A.2) was adopted for star \#3800319 due to the relatively high temperature and gravity of this star in comparison with the other stars in the sample. We analysed interactively the spectral lines. For each line the fit has been visually inspected and adjusted until reaching a satisfying solution.

\subsection{Radial velocities and the contaminating Milky Way field}

Radial velocities (see Table 1) were obtained by crosscorrelating the observed spectra with a rest frame laboratory line list using the recently released software DAOSPEC ${ }^{5}$ (Stetson and Pancino, in preparation). The final radial velocities and relative errors were computed using about 150 lines for each star. Geocentric observed radial velocities were corrected to heliocentric velocities using the IRAF task rvcorrect.

\footnotetext{
${ }^{4}$ IRAF is distributed by the National Optical Astronomy Observatories, which is operated by the association of Universities for Research in Astronomy, Inc., under contract with the National Science Foundation.

5 See http://cadcwww.hia.nrc.ca/stetson/daospec
}

The DAOSPEC code has the capability to measure the line EWs. In our case, however, we used DAOSPEC only to measure the radial velocities of the target stars while we used the IRAF task splot to measure EWs for homogeneity with our previous works on Sgr stars (Bonifacio et al. 2000, 2004). As a check, the radial velocities of a few stars have also been measured using the fxcor IRAF task for Fourier cross correlation. The radial velocities obtained using DAOSPEC and fxcor are identical, within the errors.

All but one (\#3600127, $v_{\text {helio }}=-127.6 \mathrm{~km} \mathrm{~s}^{-1}$, open circle in Fig. 2) of the 24 observed stars are indeed Sgr radial velocity members lying within $\sim 2 \sigma$ of the systemic velocity as measured by Ibata et al. (1997). In Fig. 3 we plotted the velocity distribution of the $23 \mathrm{Sgr}$ radial velocity members. The mean velocity $\left(\left\langle v_{r}\right\rangle=143.08 \pm 3.2 \mathrm{~km} \mathrm{~s}^{-1}\right)^{6}$ and the velocity dispersion of the sample $\left(\sigma=11.17 \mathrm{~km} \mathrm{~s}^{-1}\right)$ are in good agreement with the values derived by Ibata et al. (1995) and Ibata et al. (1997).

The MW model of Robin et al. (2003, hereafter R03) predicts that in the M 54 line of sight $2 \%$ of stars have $v_{r}>100 \mathrm{~km} \mathrm{~s}^{-1}$, if we consider only stars lying in the same $(V, V-K)^{7}$ selection box of the UVES sample. However, the model predicts only a $\sim 4 \%$ of giant stars $(\log g<4)$ in the selection box and none of them with $v_{r}>100 \mathrm{~km} \mathrm{~s}^{-1}$. We checked carefully the 24 stars in the sample and we are confident that all of them are indeed red giant. Therefore, even if the R03 model provides only an approximate description of the MW, there is no reason to expect any MW star among the $23 \mathrm{Sgr}$ radial velocity members in the sample.

\subsection{M-giants showing TiO molecular bands in the spectra}

The coolest (i.e. the reddest) four stars (\#2300168, \#3600181, \#3700055, \#4207391, plus symbols at $V-I>2.0$ in Fig. 2) have effective temperatures around $3600 \mathrm{~K}$ and very strong titanium oxide bands (TiO, see Selvelli \& Bonifacio 2000; Valenti et al. 1998) in the spectra (see Fig. 4). The presence of the TiO bands confirm these stars as M-giants. Such strong molecular bands prevent from a safe derivation of the equivalent widths. Therefore, we do not present the chemical analysis for these stars. In addition, stars \#3600073, \#3700178, \#3800366, \#4207953 (plus symbols at $V-I<2.0$ in Fig. 2) show weak but clearly recognizable $\mathrm{TiO}$ bands. For these stars we provide only a tentative analysis and the derived abundances will not be discussed. We plan to provide a detailed chemical analysis for these 8 stars by performing spectral synthesis including also the TiO molecular bands.

In the CMD in Fig. 2 star \#2300127 lies exactly in the region occupied by stars with $\mathrm{TiO}$ bands in their spectra. Yet this star does not present any band. The lack of the TiO molecular bands may be due to the relatively weak Fe and Ti content of this star $([\mathrm{Fe} / \mathrm{H}]=-0.81,[\mathrm{Ti} / \mathrm{Fe}]=-0.17$, see Table 3$)$.

\footnotetext{
${ }^{6}$ The quoted $3.2 \mathrm{~km} \mathrm{~s}^{-1}$ error has been estimated employing a bootstrap technique.

7 The R03 model does not provide the $(J-K)$ color, therefore we define as selection box in the $(V, V-K)$ plane the region which encloses all the target stars.
} 
Table 1. Coordinates and atmospheric parameters for the program stars.

\begin{tabular}{|c|c|c|c|c|c|c|c|c|c|c|}
\hline Star & $\alpha(J 2000.0)$ & $\delta(J 2000.0)$ & $V$ & $I$ & $(V-I)$ & $v_{\text {helio }}\left(\mathrm{km} \mathrm{s}^{-1}\right)$ & $T_{\mathrm{eff}^{a}}^{a}$ & $\log g$ & $\xi$ & {$[\mathrm{M} / \mathrm{H}]$} \\
\hline 2300127 & 185546.703 & -303524.683 & 16.09 & 14.08 & 2.01 & $+147.2 \pm 0.58$ & 3687 & 0.72 & 2.0 & -1.0 \\
\hline 2300196 & 185530.778 & -302819.635 & 16.26 & 14.54 & 1.72 & $+148.0 \pm 0.94$ & 3908 & 0.97 & 2.3 & -0.5 \\
\hline 2300215 & 185519.146 & -303027.978 & 16.52 & 14.76 & 1.76 & $+154.9 \pm 0.79$ & 3877 & 1.08 & 1.9 & -0.5 \\
\hline 2409744 & 185455.854 & -303243.106 & 16.34 & 14.54 & 1.80 & $+131.7 \pm 0.80$ & 3837 & 0.97 & 1.8 & -0.5 \\
\hline 3600230 & 185345.818 & -302549.419 & 16.43 & 14.74 & 1.69 & $+153.8 \pm 0.56$ & 3947 & 1.08 & 1.6 & -0.5 \\
\hline 3600262 & 185322.340 & -302347.172 & 16.63 & 14.88 & 1.75 & $+156.0 \pm 0.61$ & 3882 & 1.16 & 1.9 & -0.5 \\
\hline 3600302 & 185345.209 & -303055.702 & 16.65 & 14.86 & 1.79 & $+143.8 \pm 0.67$ & 3848 & 1.15 & 1.6 & -0.5 \\
\hline 3800199 & 185513.453 & -302642.249 & 15.35 & 13.90 & 1.45 & $+138.8 \pm 0.63$ & 4245 & 0.72 & 1.9 & -1.0 \\
\hline 3800204 & 18555.7440 & -302756.602 & 15.81 & 14.25 & 1.56 & $+153.2 \pm 0.61$ & 4101 & 0.93 & 2.4 & -1.5 \\
\hline 3800318 & 185458.264 & -302820.165 & 16.20 & 14.42 & 1.78 & $+151.8 \pm 0.84$ & 3856 & 0.90 & 1.9 & -0.5 \\
\hline 3800319 & 185458.088 & -302858.481 & 16.18 & 14.80 & 1.38 & $+141.1 \pm 0.88$ & 4364 & 1.23 & 1.9 & -1.5 \\
\hline 4303773 & 185402.120 & -303621.665 & 15.97 & 14.23 & 1.74 & $+143.1 \pm 0.63$ & 3895 & 0.80 & 1.9 & -0.5 \\
\hline 4304445 & 185340.606 & -303542.879 & 16.17 & 14.51 & 1.66 & $+119.9 \pm 0.59$ & 3976 & 0.95 & 1.7 & -0.5 \\
\hline 4402285 & 185319.765 & -303740.099 & 16.48 & 14.97 & 1.51 & $+159.2 \pm 0.61$ & 4156 & 1.20 & 1.5 & -0.5 \\
\hline 4408968 & 185312.886 & -303203.565 & 16.67 & 15.07 & 1.60 & $+144.0 \pm 0.68$ & 4047 & 1.26 & 2.0 & -0.5 \\
\hline $3600073^{\star}$ & 185356.477 & -302720.337 & 15.53 & 13.59 & 1.94 & $+156.2 \pm 0.67$ & 3731 & 0.41 & 2.0 & -0.5 \\
\hline $3700178^{\star}$ & 185418.068 & -302931.259 & 16.34 & 14.46 & 1.88 & $+149.1 \pm 0.81$ & 3770 & 0.92 & 2.3 & -0.5 \\
\hline $3800336^{\star}$ & 185511.635 & -302800.544 & 16.21 & 14.29 & 1.92 & $+131.1 \pm 0.74$ & 3741 & 0.83 & 2.0 & -0.5 \\
\hline $4207953^{\star}$ & 185414.546 & -303234.502 & 15.94 & 14.11 & 1.83 & $+129.0 \pm 0.89$ & 3815 & 0.74 & 1.9 & -0.5 \\
\hline $2300168^{\star}$ & 185520.010 & -302645.824 & 16.25 & 13.68 & 2.57 & $+136.0 \pm 2.81$ & 3599 & & & \\
\hline $3600181^{\star}$ & 185357.440 & -302509.207 & 16.09 & 13.90 & 2.19 & $+147.3 \pm 1.23$ & 3610 & & & \\
\hline $3700055^{\star}$ & 18542.6250 & -302648.807 & 15.57 & 13.45 & 2.12 & $+121.9 \pm 1.34$ & 3634 & & & \\
\hline $4207391^{\star}$ & 185424.849 & -303302.291 & 15.93 & 13.79 & 2.14 & $+133.7 \pm 2.01$ & 3624 & & & \\
\hline 3600127 & 185322.441 & -302359.172 & 15.94 & 14.42 & 1.52 & $-127.6 \pm 0.51$ & 4152 & & & \\
\hline
\end{tabular}

${ }^{a}$ We adopted a reddening of $E(V-I)=0.18$.

* Star showing TiO molecular bands in the spectra.

\section{Atmospheric parameters and chemical analysis}

The UVES spectra of the 19 stars for which the chemical analysis was performed (including also stars having weak TiO bands) are plotted in Fig. 5.

\subsection{Effective temperatures and surface gravities}

The effective temperatures for the target stars (see Table 1) were derived from the $(V-I)$ color assuming a reddening of $E(V-I)=0.18$ (Layden \& Sarajedini 2000) and using the calibration of Alonso et al. (1999).

We used the Girardi et al. (2002) theoretical isochrones, along with $E(V-I)=0.18$ and $(m-M)_{0}=17.10$ (Layden $\&$ Sarajedini 2000; Monaco et al. 2004) as reddening 8 and

\footnotetext{
${ }^{8}$ We assumed the same reddening for all the stars in the sample. Inspection of the Schlegel et al. (1998) reddening maps provide strong indications that there is no serious variability of extinction in the considered field (standard deviation of the reddening value: $\sigma_{E(B-V)}=0.03$, Monaco et al. 2004).
}

distance modulus, in order to estimate the gravity of the program stars. In particular, we used a $(Z=0.001$; Age $=$ 14.13 Gyr) isochrone for stars \#3800199 \#3800204 \#3800319 and a $(Z=0.008$; Age $=6.31 \mathrm{Gyr})$ isochrone for all the other stars (continuous lines in Fig. 2). These two isochrones fit into the range covered by the target stars on the CMD and the age and metallicity used are also compatible to what expected from previous works (see Monaco et al. 2002; Layden \& Sarajedini 2000; Brown et al. 1999; Bonifacio et al. 2004).

\subsection{Model atmosphere and microturbulent velocities}

For each star we computed a plane parallel model atmosphere using version 9 of the ATLAS code (Kurucz 1993) with the above atmospheric parameters. Abundances were derived from EWs using the WIDTH code (Kurucz 1993).

Microturbulent velocities $(\xi)$ were determined minimizing the dependence of the iron abundance from the $E W$, among the set of iron lines measured for each star. 
Table 2. Mean chemical abundances for the program stars. The signal to noise ratio of the coadded spectra and the number of lines used are also reported.

\begin{tabular}{|c|c|c|c|c|c|c|c|c|c|}
\hline $\operatorname{Star}^{a}$ & $\begin{array}{l}S / N \\
@ 653 \mathrm{~nm}\end{array}$ & $A(\mathrm{Fe})$ & $n$ & $A(\mathrm{Mg})$ & $n$ & $A(\mathrm{Ca})$ & $n$ & $A(\mathrm{Ti})$ & $n$ \\
\hline Sun & & 7.51 & & 7.58 & & 6.35 & & 4.94 & \\
\hline 2300127 & 20 & $6.70 \pm 0.24$ & 15 & $6.82 \pm 0.15$ & 2 & $5.35 \pm 0.10$ & 9 & $3.99 \pm 0.06$ & 7 \\
\hline 2300196 & 20 & $7.02 \pm 0.19$ & 15 & $7.03 \pm 0.18$ & 4 & $5.67 \pm 0.16$ & 9 & $4.49 \pm 0.16$ & 9 \\
\hline 2300215 & 14 & $7.28 \pm 0.18$ & 13 & $7.04 \pm 0.18$ & 4 & $5.98 \pm 0.22$ & 9 & $4.84 \pm 0.25$ & 9 \\
\hline 2409744 & 22 & $7.25 \pm 0.06$ & 10 & $7.09 \pm 0.03$ & 2 & $5.82 \pm 0.08$ & 8 & $4.67 \pm 0.13$ & 9 \\
\hline 3600230 & 21 & $7.34 \pm 0.18$ & 16 & $7.24 \pm 0.17$ & 4 & $5.81 \pm 0.14$ & 9 & $4.63 \pm 0.14$ & 9 \\
\hline 3600262 & 21 & $7.14 \pm 0.18$ & 15 & $7.27 \pm 0.13$ & 3 & $5.58 \pm 0.19$ & 9 & $4.34 \pm 0.18$ & 9 \\
\hline 3600302 & 24 & $7.20 \pm 0.18$ & 15 & $7.03 \pm 0.14$ & 4 & $5.85 \pm 0.11$ & 9 & $4.53 \pm 0.17$ & 9 \\
\hline 3800199 & 32 & $6.41 \pm 0.17$ & 15 & $6.52 \pm 0.08$ & 4 & $5.60 \pm 0.10$ & 8 & $4.32 \pm 0.11$ & 8 \\
\hline 3800204 & 31 & $5.99 \pm 0.08$ & 13 & $6.28 \pm 0.04$ & 2 & $4.97 \pm 0.07$ & 5 & $3.65 \pm 0.10$ & 8 \\
\hline 3800318 & 23 & $6.98 \pm 0.17$ & 16 & $6.83 \pm 0.15$ & 4 & $5.88 \pm 0.18$ & 9 & $4.69 \pm 0.17$ & 9 \\
\hline 3800319 & 21 & $6.14 \pm 0.26$ & 32 & $6.56 \pm 0.09$ & 2 & $5.38 \pm 0.15$ & 7 & $4.14 \pm 0.14$ & 7 \\
\hline 4303773 & 18 & $6.78 \pm 0.15$ & 14 & $6.66 \pm 0.16$ & 4 & $5.47 \pm 0.17$ & 9 & $3.98 \pm 0.05$ & 7 \\
\hline 4304445 & 33 & $7.16 \pm 0.14$ & 16 & $7.21 \pm 0.05$ & 2 & $5.76 \pm 0.21$ & 9 & $4.45 \pm 0.13$ & 9 \\
\hline 4402285 & 22 & $7.22 \pm 0.13$ & 15 & $7.11 \pm 0.17$ & 4 & $5.99 \pm 0.25$ & 9 & $4.79 \pm 0.13$ & 9 \\
\hline 4408968 & 18 & $7.09 \pm 0.16$ & 17 & $6.81 \pm 0.04$ & 3 & $5.82 \pm 0.11$ & 9 & $4.56 \pm 0.12$ & 9 \\
\hline $3600073^{\star}$ & 43 & $6.73 \pm 0.18$ & 17 & $6.67 \pm 0.14$ & 4 & $5.23 \pm 0.15$ & 9 & $3.87 \pm 0.20$ & 9 \\
\hline $3700178^{\star}$ & 19 & $7.06 \pm 0.19$ & 16 & $6.83 \pm 0.17$ & 3 & $5.47 \pm 0.32$ & 9 & $4.16 \pm 0.24$ & 9 \\
\hline $3800336^{\star}$ & 25 & $6.88 \pm 0.16$ & 14 & $7.01 \pm 0.10$ & 3 & $5.63 \pm 0.12$ & 8 & $4.40 \pm 0.16$ & 9 \\
\hline $4207953^{\star}$ & 30 & $7.10 \pm 0.13$ & 15 & $6.97 \pm 0.10$ & 4 & $5.63 \pm 0.19$ & 9 & $4.51 \pm 0.17$ & 8 \\
\hline
\end{tabular}

* Star showing TiO molecular bands in the spectra. $A(\mathrm{X})=\log \left(\frac{X}{H}\right)+12.00$.

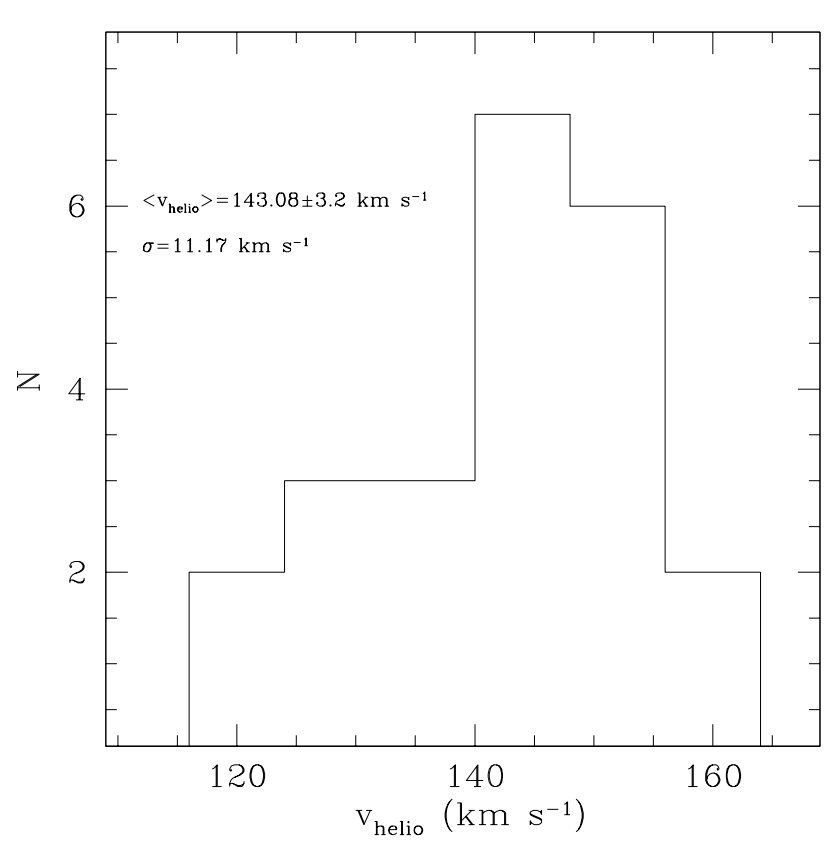

Fig. 3. Heliocentric radial velocity distribution for the 23 programme stars Sgr radial velocity members.
In Fig. 6 we plotted $\xi$ as a function of the adopted gravity for the stars studied by Ivans et al. (2001, bottom panel, hereafter I01), Shetrone et al. (2003, middle panel, hereafter S03) and for stars in our sample (top panel). A clear trend is present in both the I01 and S03 samples. The same trend is present also in our sample, albeit with a larger scatter. Continuous lines are least square fits to the data points. In the case of our sample the fit was obtained excluding the points having the highest and lowest $\xi\left(2.7\right.$ and $\left.0.6 \mathrm{~km} \mathrm{~s}^{-1}\right)$ and the point with the lowest surface gravity $(\log g=0.41)$. As can be seen, the three fitting lines are very similar to each other. A weak dependence of the $\xi$ from the effective temperature was found and it can be safely neglected as a first order approximation. For stars \#2300127, \#2300215 and \#3800319 (filled circles in Fig. 6) the $\xi$ is 2.7, 2.5 and $0.6 \mathrm{~km} \mathrm{~s}^{-1}$, respectively, i.e. more than $2-\sigma$ far from the fitting relation. When working with low $S / N$, highly crowded spectra, it is difficult to measure weak Fe lines accurately. This may lead to incorrect $\xi$. Thus, for stars \#2300127, \#2300215 and \#3800319 we adopted the value obtained from the fitting relation $\xi=-0.35 \times \log g+2.29$, i.e. $\xi=2.0,1.9$ and $1.9 \mathrm{~km} \mathrm{~s}^{-1}$, respectively. 


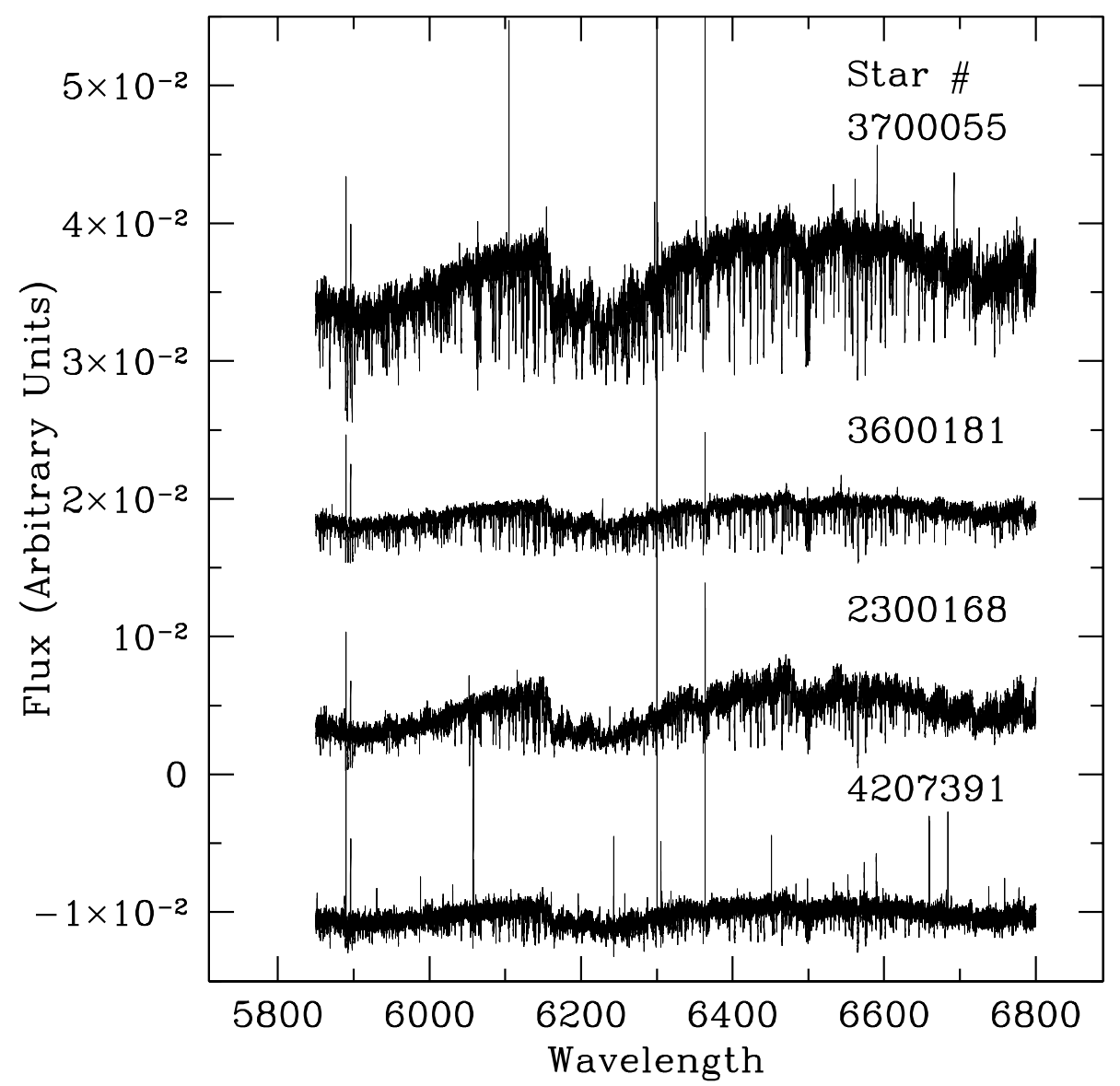

Fig. 4. Coadded UVES spectra of the 4 Sgr M-giants having strong TiO bands.

\subsection{Chemical abundances}

The atmospheric parameters $\left(T_{\text {eff }}, \log g, \xi\right.$ and the assumed global metallicity $[\mathrm{M} / \mathrm{H}]$ ) adopted for the program stars are reported in Table 1. The chemical abundances obtained for each line are reported in Tables A.1 and A.2. The mean and standard deviation of such abundances are reported in Tables 2 and 3 (as $[\mathrm{X} / \mathrm{H}]$ abundances in the latter case) for each chemical species. In Table 2 we also reported the number of lines used to obtain the mean abundance for each species. The line scatter reported in Table 2 should be representative of the statistical error arising from the noise in the spectra and from uncertainties in the measurement of the equivalent widths.

Under the assumption that each line provides an independent measure of the abundance, the error in the mean abundances should be obtained by dividing the line scatter by $\sqrt{n}$ (where $n$ is the number of measured lines) and by adding to this figure the errors arising from the uncertainties in the atmospheric parameters. In Table 4 we report these latter errors in the case of star \#3800318, taken as representative of the whole sample.

In Fig. 7 we plotted the metallicity distribution obtained. Our sample spans a rather large metallicity range $(-1.52 \leq$ $[\mathrm{Fe} / \mathrm{H}] \leq-0.17)$. The distribution peaks around $[\mathrm{Fe} / \mathrm{H}] \simeq-0.4$ and presents an extended metal poor tail ${ }^{9}$. In particular, considering only stars more metal rich than $[\mathrm{Fe} / \mathrm{H}] \simeq-1$, which should be representative of the Sgr dominant population, we obtain a mean value of $\langle[\mathrm{Fe} / \mathrm{H}]\rangle=-0.41 \pm 0.20$.

In Fig. 8 we plotted the $[\mathrm{Ti} / \mathrm{Fe}],[\mathrm{Ca} / \mathrm{Fe}]$ and $[\mathrm{Mg} / \mathrm{Fe}]$ ratios (from top to bottom panel) for the program stars as a function of the $[\mathrm{Fe} / \mathrm{H}]$ abundance. The 5 M 54 stars studied by Brown et al. (1999, hereafter B99) are plotted as large open stars. Assuming $\langle[\alpha / \mathrm{Fe}]\rangle=\frac{[\mathrm{Mg} / \mathrm{Fe}]+[\mathrm{Ca} / \mathrm{Fe}]}{2}$, we also obtain a mean value of $\langle[\alpha / \mathrm{Fe}]\rangle=-0.17 \pm 0.07$ for the dominant population.

Following Salaris et al. (1993), these values correspond to a global metallicity ${ }^{10}$ of $[\mathrm{M} / \mathrm{H}]=-0.51$, which is in good agreement with the recent photometric estimate by Monaco et al. (2002).

\subsection{Notes on metal poor stars: \#3800199, \#3800204,\#3800319}

The three most metal-poor stars (\#3800199 \#3800204 and \#3800319) occupy in the optical CMD (see Fig. 2)

\footnotetext{
${ }^{9}$ Preliminary results obtained from the GIRAFFE sample show that such tail extends at least down to $[\mathrm{Fe} / \mathrm{H}]<-2.5$ (Zaggia et al. 2004; Bonifacio et al. 2005).

10 The "global metallicity" is defined as: $[\mathrm{M} / \mathrm{H}]=[\mathrm{Fe} / \mathrm{H}]+$ $\log \left(0.638 \times 10^{[\alpha / \mathrm{Fe}]}+0.362\right)$.
} 


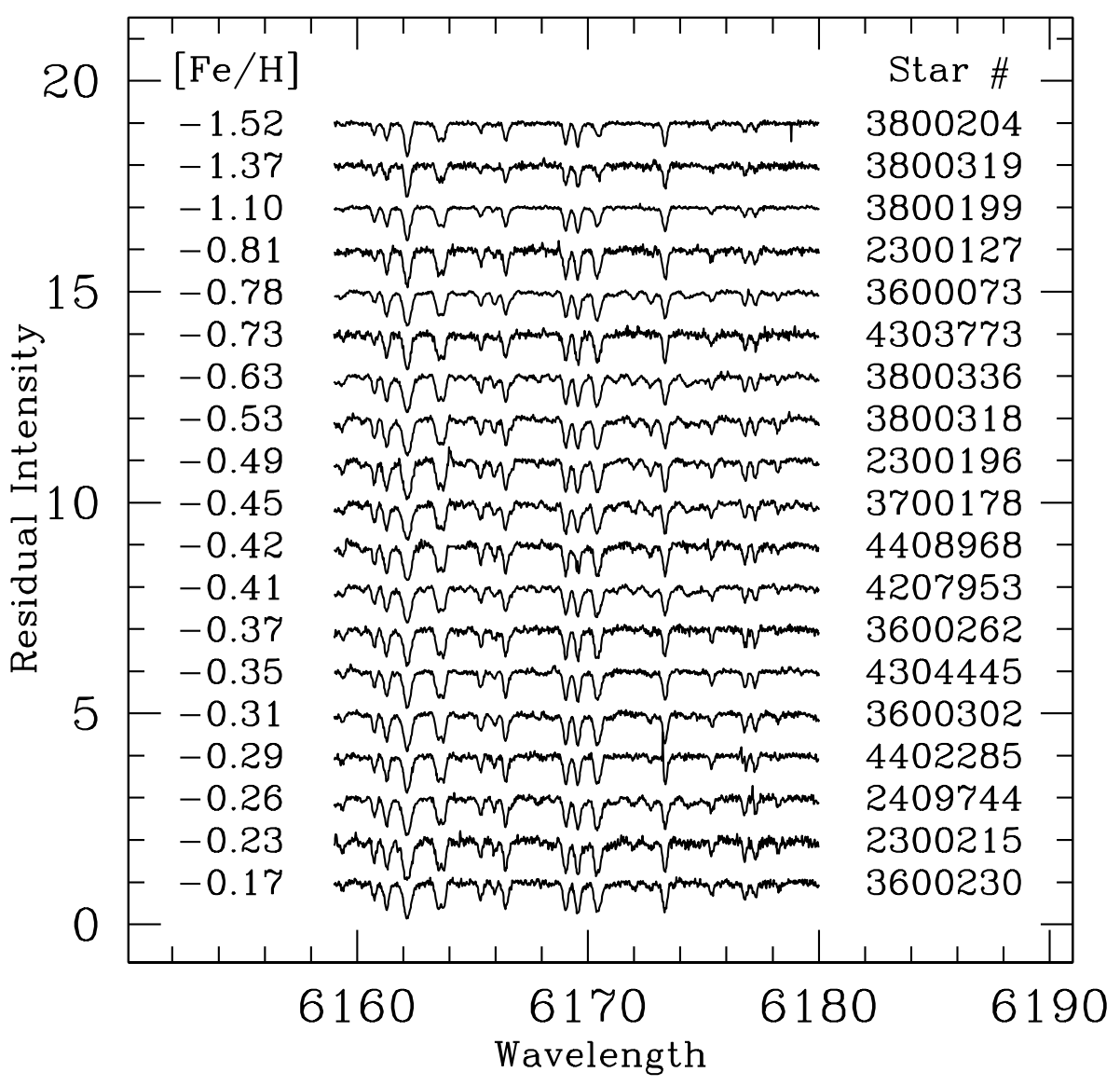

Fig. 5. Coadded UVES spectra of the 19 Sgr giants analyzed in this paper. Labels on the right denote the star number, those on the left the [Fe/H].

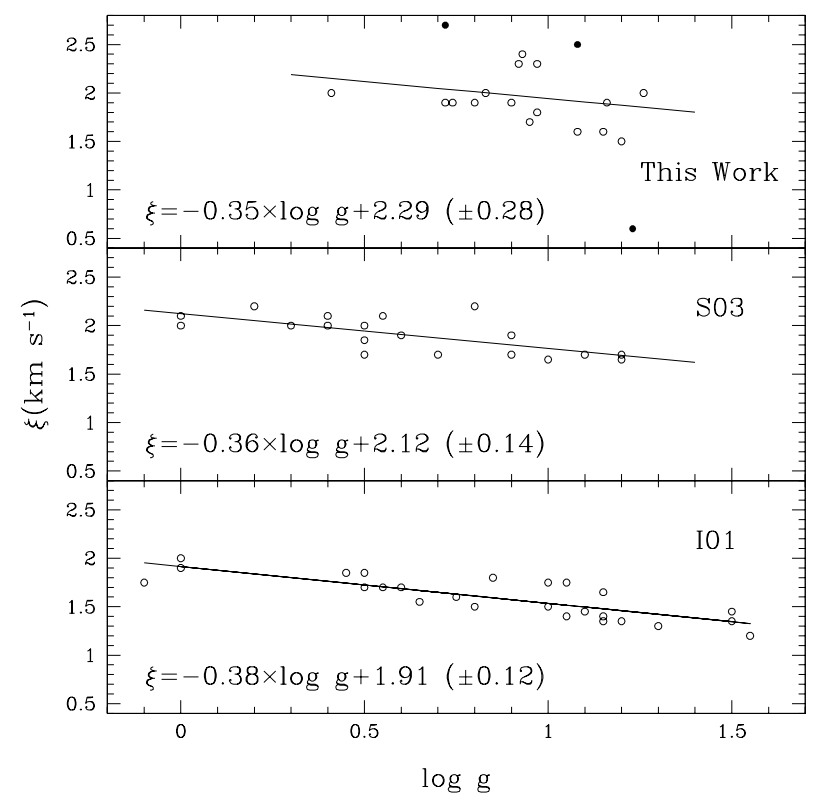

Fig. 6. Microturbulent velocities as a function of the surface gravity for the programme stars (top panel) and stars in the S03 and I01 samples (middle and bottom panel respectively). Least square fits to the data are plotted as continuous lines. The filled circles in the upper panel mark stars more than $2-\sigma$ far from the fitting relation.

positions compatible with the M 54 RGB (which is roughly represented by the bluer isochrone in the plot).
The most metal-poor star $(\# 3800204,[\mathrm{Fe} / \mathrm{H}]=-1.52)$ lies very near to the M 54 center $\left(\sim 1^{\prime}\right)$ and its chemical abundances (Fig. 8) are identical to those of the M 54 stars studied by B99. Therefore, it seems quite likely that this star does indeed belong to M 54.

Star \#3800319 $([\mathrm{Fe} / \mathrm{H}]=-1.37)$ is also quite near $(\sim 1.4)$ to the cluster center but its chemical composition is only marginally compatible with M 54 and it will be considered a Sgr field star. However, we note that Layden \& Sarajedini (2000) claimed a metallicity dispersion of $\sim 0.16$ dex for M 54 from the width of the red giant branch.

Star \#3800199 $([\mathrm{Fe} / \mathrm{H}]=-1.10)$ is placed at 3.2 from the cluster center (which corresponds to $\sim 7$ half light radii, Trager et al. 1995) and is significantly more metal rich than M 54 $([\mathrm{Fe} / \mathrm{H}] \sim-1.55, \mathrm{~B} 99)$. Therefore, we consider star \#3800199 part of the Sgr galaxy field.

\section{Comparison with previous works}

Beside the present work, chemical abundances have been presented for Sgr RGB stars by Bonifacio et al. (2000, 2004, 2 and 10 stars, respectively) and by Smecker-Hane \& McWilliam (2002, hereafter S02, 14 stars). Bonifacio et al. (2004, hereafter B04) also considered the two stars studied in Bonifacio et al. (2000) obtaining a final sample of 12 stars.

In Fig. 9 we plotted the $[\alpha / \mathrm{Fe}]$ as a function of the iron abundance for the stars in the 3 samples. Stars in our sample are 
Table 3. Mean abundance ratios for the program star.

\begin{tabular}{lcrrr}
\hline \hline Star & {$[\mathrm{Fe} / \mathrm{H}]$} & {$[\mathrm{Mg} / \mathrm{Fe}]$} & {$[\mathrm{Ca} / \mathrm{Fe}]$} & {$[\mathrm{Ti} / \mathrm{Fe}]$} \\
\hline 2300127 & $-0.81 \pm 0.24$ & +0.05 & -0.19 & -0.14 \\
2300196 & $-0.49 \pm 0.19$ & -0.06 & -0.19 & +0.04 \\
2300215 & $-0.23 \pm 0.18$ & -0.31 & -0.14 & +0.13 \\
2409744 & $-0.26 \pm 0.06$ & -0.23 & -0.27 & -0.01 \\
3600230 & $-0.17 \pm 0.18$ & -0.17 & -0.37 & -0.14 \\
3600262 & $-0.37 \pm 0.18$ & +0.06 & -0.40 & -0.23 \\
3600302 & $-0.31 \pm 0.18$ & -0.24 & -0.19 & -0.10 \\
3800199 & $-1.10 \pm 0.17$ & +0.04 & +0.35 & +0.48 \\
3800204 & $-1.52 \pm 0.08$ & +0.22 & +0.14 & +0.23 \\
3800318 & $-0.53 \pm 0.17$ & -0.22 & +0.06 & +0.28 \\
3800319 & $-1.37 \pm 0.26$ & +0.35 & +0.40 & +0.57 \\
4303773 & $-0.73 \pm 0.15$ & -0.19 & -0.15 & -0.23 \\
4304445 & $-0.35 \pm 0.14$ & -0.02 & -0.24 & -0.14 \\
4402285 & $-0.29 \pm 0.13$ & -0.18 & -0.07 & +0.14 \\
4408968 & $-0.42 \pm 0.16$ & -0.35 & -0.11 & +0.04 \\
& & & & \\
$3600073^{\star}$ & $-0.78 \pm 0.18$ & -0.13 & -0.34 & -0.29 \\
$3700178^{\star}$ & $-0.45 \pm 0.19$ & -0.30 & -0.43 & -0.33 \\
$3800336^{\star}$ & $-0.63 \pm 0.16$ & +0.06 & -0.09 & +0.09 \\
$4207953^{\star}$ & $-0.41 \pm 0.13$ & -0.20 & -0.31 & -0.02 \\
\hline
\end{tabular}

* Star showing TiO molecular bands in the spectra $[\mathrm{X} / \mathrm{Y}]=\log \left(\frac{X}{Y}\right)-$ $\log \left(\frac{X}{Y}\right)_{\odot}$.

Table 4. Errors in the abundances of star \# 3800318 due to uncertainties in the atmospheric parameters.

\begin{tabular}{lrrrr}
\hline \hline & $\Delta A(\mathrm{Fe})$ & $\Delta A(\mathrm{Mg})$ & $\Delta A(\mathrm{Ca})$ & $\Delta A(\mathrm{Ti})$ \\
\hline$\Delta \xi= \pm 0.2 \mathrm{~km} \mathrm{~s}^{-1}$ & -0.08 & $\mp 0.04$ & $\mp 0.12$ & -0.15 \\
& +0.10 & & & +0.17 \\
& & & & \\
$\Delta T_{\text {eff }}= \pm 100 \mathrm{~K}$ & -0.04 & -0.02 & \pm 0.10 & ${ }_{-0.12}^{+0.13}$ \\
& +0.07 & +0.05 & & \\
& & & & \\
$\Delta \log g= \pm 0.50$ & ${ }^{+0.16}$ & ${ }_{-0.07}^{+0.08}$ & -0.04 & +0.05 \\
\hline
\end{tabular}

plotted as filled circles, while stars in the B04 and S02 sample are plotted as empty squares and empty triangles, respectively. The 5 M 54 stars studied by B99 are marked as large open stars. The $\alpha$ element abundance ratio is defined as $[\alpha / \mathrm{Fe}]=$ $\frac{[\mathrm{Mg} / \mathrm{Fe}]+[\mathrm{Ca} / \mathrm{Fe}]}{2}$ for stars in our sample and in the B04 and B99 samples, while it is defined as $[\alpha / \mathrm{Fe}]=\frac{[\mathrm{Si} / \mathrm{Fe}]+[\mathrm{Ca} / \mathrm{Fe}]+[\mathrm{Ti} / \mathrm{Fe}]}{3}$ for stars in the S02 sample ${ }^{11}$.

Stars in the S02 sample range from $[\mathrm{Fe} / \mathrm{H}] \simeq-1.6$ to $[\mathrm{Fe} / \mathrm{H}] \simeq 0$. In particular, 3 stars in their sample have $[\mathrm{Fe} / \mathrm{H}]<$ -1 and 11 stars are in the range $-0.7 \div 0.0$. This latter subsample has a mean metallicity and $\alpha$ element abundance ratio of: $\langle[\mathrm{Fe} / \mathrm{H}]\rangle=-0.36 \pm 0.19$ and $\langle[\alpha / \mathrm{Fe}]\rangle=+0.01 \pm 0.04$. However, it is important to note that these values should not be considered as representative of the dominant population, since

\footnotetext{
${ }^{11}$ S02 do not provide abundances for each species but only mean values.
}

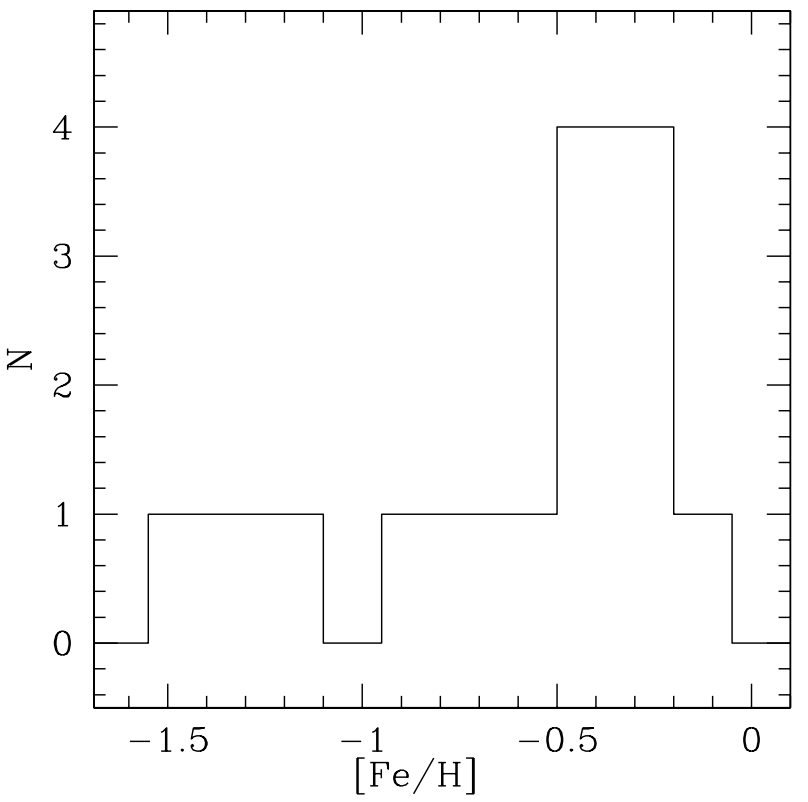

Fig. 7. Metallicity distribution of the program stars.

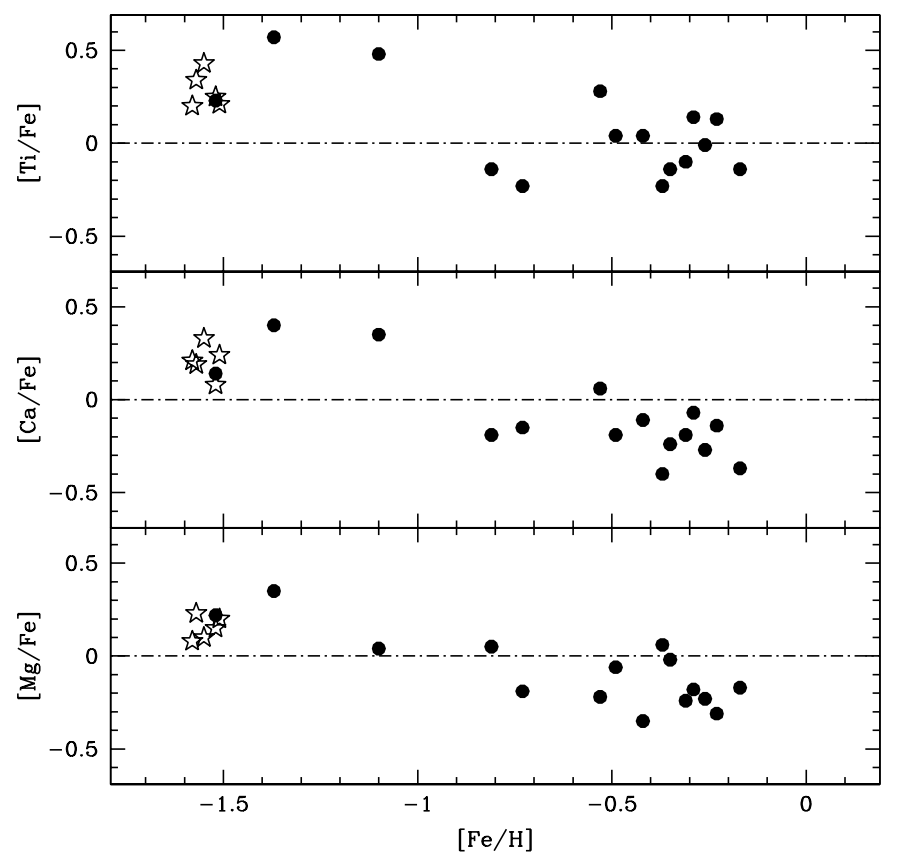

Fig. 8. $\alpha$ element abundance ratios $([\mathrm{Ti} / \mathrm{Fe}],[\mathrm{Ca} / \mathrm{Fe}]$ and $[\mathrm{Mg} / \mathrm{Fe}]$ from top to bottom panel) as a function of the iron abundance for the program stars. Large open stars mark the 5 M 54 stars studied by B99.

their target selection has been biased toward stars with metallicities within 0.5 dex of the solar value based on previously obtained approximate metallicities (McWilliam \& Smecker-Hane 2004).

The metallicity range of stars in the B04 sample, on the other hand, is $-0.83 \leq[\mathrm{Fe} / \mathrm{H}]<+0.09$. Therefore, it extends toward slightly higher metallicity with respect to the S02 sample, but it lacks of metal-poor stars. The mean metallicity and $\alpha$ element abundance ratio of the B04 sample are: $\langle[\mathrm{Fe} / \mathrm{H}]\rangle=-0.23 \pm 0.26$ and $\langle[\alpha / \mathrm{Fe}]\rangle=-0.20 \pm 0.06$. 


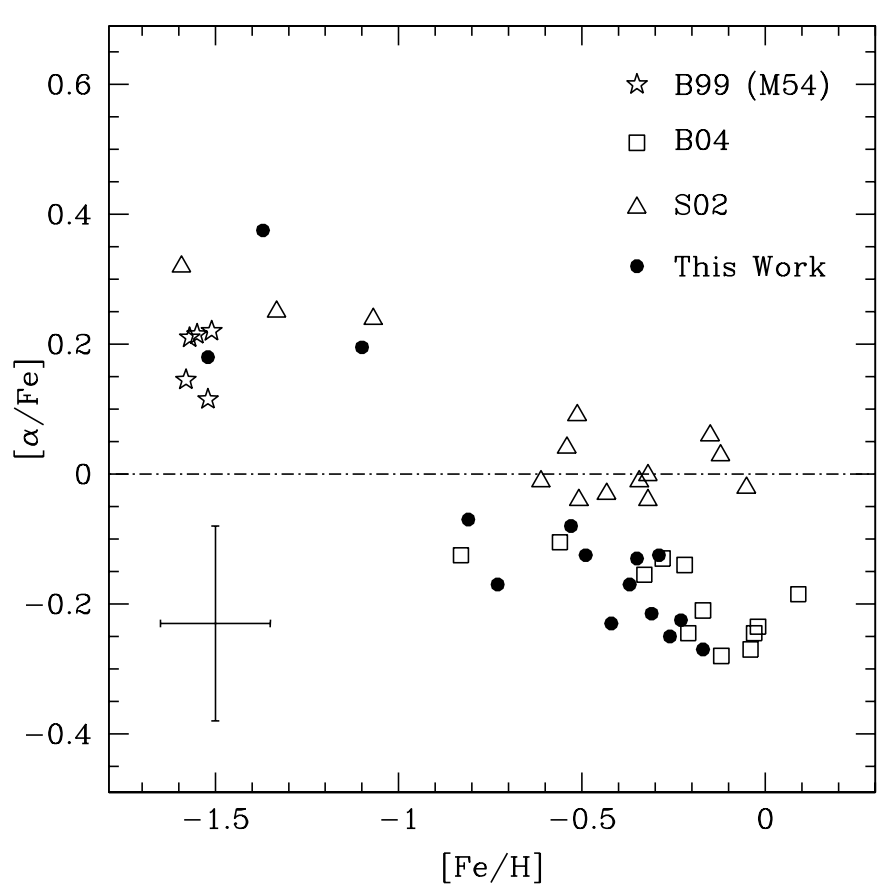

Fig. 9. $\alpha$ element abundance ratio - defined as: $[\alpha / \mathrm{Fe}]=\frac{[\mathrm{Mg} / \mathrm{Fe}]+[\mathrm{Ca} / \mathrm{Fe}]}{2}$ - as a function of the iron abundance for the program stars (filled circles). Large open stars mark the 5 M 54 stars studied by B99. Open squares and open triangles mark stars in the B04 and S02 samples, respectively. For stars in the S02 sample: $[\alpha / \mathrm{Fe}]=\frac{[\mathrm{Si} / \mathrm{Fe}]+[\mathrm{Ca} / \mathrm{Fe}]+[\mathrm{Ti} / \mathrm{Fe}]}{3}$.

The mean iron abundance obtained in this paper $([\mathrm{Fe} / \mathrm{H}]=$ -0.41 ) is similar to that of the S02 and B04 samples. The 0.18 dex difference between the B04 mean iron abundance and our figure would be also a little bit lowered (by $0.06 \div 0.09 \mathrm{dex}$ ) by taking into account the different assumption about the reddening (B04 adopted $E(V-I)=0.22$ from Marconi et al. 1998). The different target selection criterion adopted by B04 may also be responsible for the residual difference in the mean iron abundance $(\sim 0.1 \mathrm{dex})$, which is, nevertheless, well inside the involved errors.

The $\langle[\alpha / \mathrm{Fe}]\rangle$ ratio obtained by B04 is very similar to our value $\left(\langle[\alpha / \mathrm{Fe}]\rangle=\frac{[\mathrm{Mg} / \mathrm{Fe}]+[\mathrm{Ca} / \mathrm{Fe}]}{2}=-0.17\right)$. S02 evaluate the $\alpha$ element abundance ratio as $[\alpha / \mathrm{Fe}]=\frac{[\mathrm{Si} / \mathrm{Fe}]+[\mathrm{Ca} / \mathrm{Fe}]+[\mathrm{Ti} / \mathrm{Fe}]}{3}$. Considering $[\alpha / \mathrm{Fe}]=\frac{[\mathrm{Ca} / \mathrm{Fe}]+[\mathrm{Ti} / \mathrm{Fe}]}{2}$, we obtain $\mathrm{a}\langle[\alpha / \mathrm{Fe}]\rangle$ fairly similar to the S02 figure. The small residual difference ( $\sim 0.1$ dex higher in the S02 sample) may be partly ascribed to the $[\mathrm{Si} / \mathrm{Fe}]$ abundances and, possibly, to a different set of lines and atomic parameters adopted in the chemical analysis. Unfortunately, S02 neither provide abundances for each species nor the atomic data and the adopted line list and this hypothesis cannot be checked further.

Finally, as already stressed in Sect. 3.4, we remark that the $\mathrm{Fe}, \mathrm{Mg}, \mathrm{Ca}$ and $\mathrm{Ti}$ abundances of star \#3800204 are consistent with the results obtained by B99 for M 54 stars.

\section{Discussion and conclusions}

The main purpose of this paper was to study the chemical composition of the dominant population of the Sgr dSph galaxy. We selected 24 target stars using the 2 MASS infrared CMD, where the upper RGB of Sgr is well separated from the MW field.
Target stars have been observed using the red arm of the high resolution spectrograph FLAMES-UVES. We reported radial velocities for these 24 stars and all but one are Sgr radial velocity members. Eight stars show strong or visible $\mathrm{TiO}$ bands. For stars with weak TiO bands we present a tentative chemical analysis while we do not present any chemical analysis for stars presenting strong $\mathrm{TiO}$ bands in the spectra.

For the remaining 15 stars, we reported $\mathrm{Fe}, \mathrm{Mg}, \mathrm{Ca}$ and Ti chemical abundances. This is the largest sample of high resolution spectra analyzed so far for stars in the Sgr dSph galaxy, and the only sample thoroughly representative of the Sgr dominant population.

The metallicity ranges from $[\mathrm{Fe} / \mathrm{H}]=-1.52$ to $[\mathrm{Fe} / \mathrm{H}]=$ -0.17 . Three stars have $[\mathrm{Fe} / \mathrm{H}]<-1$ and the most metal poor of them (\#3800204) can be reasonably considered M 54 member.

The mean iron content of stars with $[\mathrm{Fe} / \mathrm{H}]>-1$ (i.e. the Sgr dominant population) is $\langle[\mathrm{Fe} / \mathrm{H}]\rangle=-0.41 \pm 0.20$, with a mean $\alpha$ element abundance ratio $\langle[\alpha / \mathrm{Fe}]\rangle=-0.17 \pm 0.07$. These figures lead to a global metallicity $[\mathrm{M} / \mathrm{H}]=-0.51$ which is in close agreement with the most recent photometric estimates obtained for the Sgr dominant population (Monaco et al. 2002).

In order to obtain a more statistically significant sample, we now join the B04 and our samples. In Fig. 10 we plotted in the $[\alpha / \mathrm{Fe}]$ vs. $[\mathrm{Fe} / \mathrm{H}]$ plane the mean points obtained for Sgr from this larger sample of Sgr stars as filled circles. For $-0.65<[\mathrm{Fe} / \mathrm{H}]<0.1$, filled circles represent running means with 0.20 dex as bin and 0.1 dex as step. For stars having $-1.0<[\mathrm{Fe} / \mathrm{H}]<-0.65$ and $-1.5<[\mathrm{Fe} / \mathrm{H}]<-1$ (i.e. excluding star \#3800204 which has been tagged as M 54 member) filled circles are straight means of the $[\mathrm{Fe} / \mathrm{H}]$ and $[\alpha / \mathrm{Fe}]$ with the corresponding standard deviations as errorbars. A weak, but clearly recognizable trend between the $\alpha$ element abundance ratio and the mean iron abundance exists at high metallicity. Such a trend waits to be confirmed from a much more extended sample such as that obtained using the FLAMES-GIRAFFE multifibre spectrograph which is currently under analysis. For $[\mathrm{Fe} / \mathrm{H}]<-1$, a sudden increase of the $[\alpha / \mathrm{Fe}]$ is apparent.

The mean $[\alpha / \mathrm{Fe}]$ at low metallicities is consistent with the values observed in MW stars (crosses in Fig. 10, from Venn et al. 2004) of comparable metallicities and somewhat higher with respect to stars in the LG galaxies (asterisks in the figure, from Venn et al. 2004). Therefore, metal-poor stars lost in early passages which now are not recognizable as Sgr tidal debris (Helmi 2004), would be part of the typical content of the MW Halo and impossible to tag as an accreted component from the chemical composition.

The three metal-poor stars in the S02 sample are compatible with MW stars as well. This occurrence led the authors to suggest that the upper mass end of the Sgr initial mass function (IMF) should not be significantly different from the MW one. The level of $[\alpha / \mathrm{Fe}]$ which characterizes a galaxy at low metallicities may indeed give information on the IMF of the galaxy at that time (see McWilliam 1997, and references therein), since the amount of $\alpha$ elements and iron produced by a type II SN is a function of the mass of the SN progenitor. Although this is true in principle, in practice this information may not be presently 


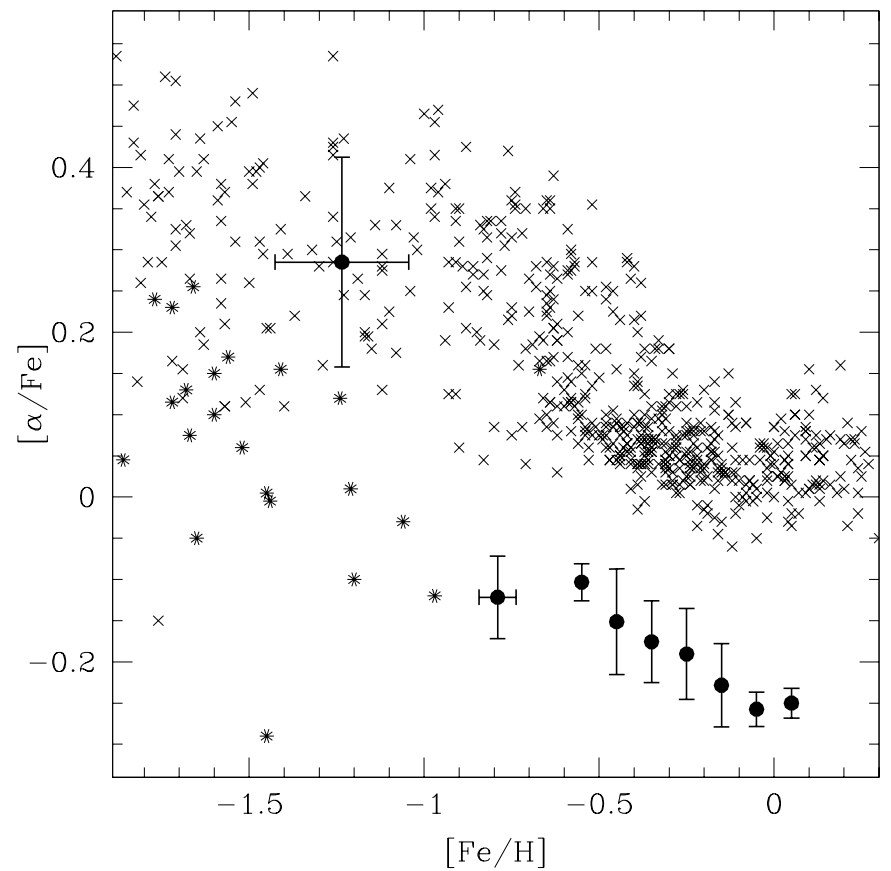

Fig. 10. $[\alpha / \mathrm{Fe}]=\frac{[\mathrm{Mg} / \mathrm{Fe}]+[\mathrm{Ca} / \mathrm{Fe}]}{2}$ as a function of $[\mathrm{Fe} / \mathrm{H}]$ for stars in the MW and in Local Group dwarf galaxies (crosses and asterisks, respectively, from Venn et al. 2004). Filled circles are mean points for Sgr obtained joining the B04 sample and our data.

extracted. In fact the ratio of the $\alpha$ elements and iron produced by a type II SN is also a sensitive function of the "mass cut", i.e. the mass coordinate which separates the material of the SN which "falls back" on the SN remnant from the material which is ejected. The deeper the mass cut, the more iron-peak elements are ejected, thus lowering the overall $[\alpha / \mathrm{Fe}]$. Current $\mathrm{SN}$ models are unable to determine the mass cut in a self consistent way or from first principles: the mass cut is always assumed. We do not have either any indication on whether the mass cut is in any sense "Universal" or if it may vary e.g. depending on the mass of the star or on its metallicity. With this state of affairs, any inference on the IMF from the level of the $[\alpha / \mathrm{Fe}]$ ratio of a galaxy would be highly uncertain.

The metal-rich Sgr stars lie on the extension to high metallicity of the pattern followed by stars in LG galaxies and below MW stars. A low $[\alpha / \mathrm{Fe}]$ at high metallicity is traditionally interpreted as evidence for a slow or bursting star formation rate (S02, B04, Marconi et al. 1994). On the contrary, in order to reproduce the $\operatorname{Sgr}[\alpha / \mathrm{Fe}]$ ratios, Lanfranchi $\&$ Matteucci (2003) required a high star formation rate. However, they constrained their model using preliminary abundances presented by Smecker-Hane \& McWilliam (1999). The somewhat lower $[\alpha / \mathrm{Fe}]$ values obtained here and in S02 and B04 should be in better agreement with a lower star formation rate.

Our data suggest that Sgr had a different chemical evolution from both the MW and the LG galaxies (see Fig. 10). A different chemical evolution for Sgr with respect to the other LG galaxies is expected, since Sgr experienced strong and disruptive dynamical interactions with the MW. Such interactions are witnessed by the Sgr tidal tails studied by Majewski et al. (2003) and are expected to trigger star formation activity (see, for instance, Kravtsov et al. 2004; Mayer et al. 2001; Zaritsky \& Harris 2004).

Finally, we note that the Large Magellanic Cloud (LMC) metallicity distribution strongly resembles the Sgr one. In fact, Cole et al. (2005) approximated the metallicity distribution of the $\mathrm{LMC}$ bar by two Gaussians having $[\mathrm{Fe} / \mathrm{H}]=-0.37 \pm 0.15$ and $[\mathrm{Fe} / \mathrm{H}]=-1.08 \pm 0.46$ and containing $89 \%$ and $11 \%$ of the stars, respectively. The same results hold also for the LMC disk (see Cole et al. 2005). Clearly, Sgr has the same mean metallicity of the LMC dominant population as well as the same fraction of metal-poor stars (see Monaco et al. 2003). Such occurrence may suggest a similarity of the Sgr progenitor with the LMC.

Acknowledgements. Part of the data analysis has been performed using software developed by P. Montegriffo at the INAF - Osservatorio Astronomico di Bologna. This research was done with support from the Italian MIUR COFIN/PRIN grants 2002028935 and 2004025729. We are grateful to L. Girardi for useful comments and to G. Schiulaz for a careful reading of the manuscript.

\section{References}

Alonso, A., Arribas, S., \& Martínez-Roger, C. 1999, A\&AS, 140, 261 Bellazzini, M., Ferraro, F. R., \& Buonanno, R. 1999b, MNRAS, 307, 619

Bonifacio, P., et al. 2004, Proc. of the ESO/Arcetri Conference on Chemical Abundances and Mixing in Stars in the Milky Way and its Satellites held in Castiglione della Pescaia, Italy, 13-17 September Bonifacio, P., Hill, V., Molaro, P., et al. 2000, A\&A, 359, 663

Bonifacio, P., Sbordone, L., Marconi, G., Pasquini, L., \& Hill, V. 2004, A\&A, 414, 503

Brown, J. A., Wallerstein, G., \& Gonzalez, G. 1999, AJ, 118, 1245

Bullock, J. S., \& Johnston, K. V. 2004, Proc. of the conference Satellites and Tidal Streams, held in La Palma, Spain, 26-30 May 2003, ed. F. Prada, D. Martinez-Delgado, \& T. Mahoney, in press [arXiv:astro-ph/0401625]

Cole, A. A. 2001, ApJ, 559, L17

Cole, A. A., Tolstoy, E., Gallagher, J. S., \& Smecker-Hane, T. A. 2005, AJ, 129, 1465

Ferraro, F. R., D'Amico, N., Possenti, A., Mignani, R. P., \& Paltrinieri, B. 2001, ApJ, 561, 337

Fulbright, J. P., Rich, R. M., \& Castro, S. 2004, ApJ, 612, 447

Fuhr, J. R., Martin, G. A., \& Wiese, W. L. 1988, J. Phys. Chem. Ref. Data, 17, Suppl. 4 (FMW)

Geisler, D., Smith, V. V., Wallerstein, G., Gonzalez, G., \& Charbonnel, C. 2005 , AJ, 129, 1428

Girardi, L., Bertelli, G., Bressan, A., et al. 2002, A\&A, 391, 195

Gratton, R. G., Carretta, E., Claudi, R., Lucatello, S., \& Barbieri, M. 2003, A\&A, 404, 187 (G03)

Helmi, A. 2004, MNRAS, 351, 643

Ibata, R. A., Gilmore, G., \& Irwin, M. J. 1994, Nature, 370, 194

Ibata, R. A., Gilmore, G., \& Irwin, M. J. 1995, MNRAS, 277, 781

Ibata, R. A., Wyse, R. F. G., Gilmore, G., Irwin, M. J., \& Suntzeff, N. B. 1997, AJ, 113, 634

Ivans, I. I., Kraft, R. P., Sneden, C., et al. 2001, AJ, 122, 1438

Kravtsov, A. V., Gnedin, O. Y., \& Klypin, A. A. 2004, ApJ, 609, 482

Kurucz, R. L. 1993, CD-ROM 13, 18

http://kurucz . harvard.edu

Layden, A. C., \& Sarajedini, A. 2000, AJ, 119, 1760 (LS00)

Lanfranchi, G. A., \& Matteucci, F. 2003, MNRAS, 345, 71 
Majewski, S. R., Skrutskie, M. F., Weinberg, M. D., \& Ostheimer, J. C. 2003, ApJ, 599, 1082

Majewski, S. R., Kunkel, W. E., Law, D. R., et al. 2004, AJ, 128, 245

Marconi, G., Matteucci, F., \& Tosi, M. 1994, MNRAS, 270, 35

Marconi, G., Buonanno, R., Castellani, M., et al. 1998, A\&A, 330, 453

Martin, G. A., Fuhr, J. R., \& Wiese, W. L. 1988, J. Phys. Chem. Ref. Data, 17, Suppl. 3 (MFW)

Mayer, L., Governato, F., Colpi, M., et al. 2001, ApJ, 559, 754

Mateo, M., Udalski, A., Szymansky, M., et al. 1995, AJ, 110, 1141

McWilliam, A. 1997, ARA\&A, 35, 503

Mc William, A., \& Smecker-Hane, T. 2004, ASP Conf. on Cosmic Abundances as Records of Stellar Evolution and Nucleosynthesis, 2005, ed. F. N. Bash, \& T. G. Barnes [arXiv: astro-ph/0409083]

Monaco, L., Ferraro, F. R., Bellazzini, M., \& Pancino, E. 2002, ApJ, 578, L47

Monaco, L., Bellazzini, M., Ferraro, F. R., \& Pancino, E. 2003, ApJ, 597, L25

Monaco, L., Bellazzini, M., Ferraro, F. R., \& Pancino, E. 2004, MNRAS, 353, 874

Monaco, L., Bellazzini, M., Ferraro, F. R., \& Pancino, E. 2005, MNRAS, 356, 1396

O'Brian, T. R., Wickliffe, M. E., Lawler, J. E., Whaling, J. W., \& Brault, W. 1991, Opt. Soc. Am. J. B Opt. Phys., 8, 1185 (O)

Pasquini, L., Avila, G., Allaert, E., et al. 2000, Proc. SPIE, 4008, 129

Robertson, B., Bullock, J. S., Font, A. S., Johnston, K. V., \& Hernquist, L. 2005, ApJ, submitted [arXiv: astro-ph/0501398]
Robin, A. C., Reylé, C., Derrière, S., \& Picaud, S. 2003, A\&A, 409, 523

Salaris, M., Chieffi, A., \& Straniero, O. 1993, ApJ, 414, 580

Schlegel, D. J., Finkbeiner, D. P., \& Davis, M. 1998, ApJ, 500, 525

Selvelli, P. L., \& Bonifacio, P. 2000, A\&A, 364, L1

Shetrone, M. D., Bolte, M., \& Stetson, P. B. 1998, AJ, 115, 1888

Shetrone, M. D., Côté, P., \& Sargent, W. L. W. 2001, ApJ, 548, 592

Shetrone, M., Venn, K. A., Tolstoy, E., et al. 2003, AJ, 125, 684

Smecker-Hane, T., \& Mc William, A. 1999, ASP Conf. Ser., 192, 150

Smecker-Hane, T. A., \& McWilliam, A. 2002, ApJ, submitted [arXiv:astro-ph/0205411]

Smith, G., \& Raggett, D. S. J. 1981, Journal of Physics B Atomic Molecular Physics, 14, 4015 (SR)

Tolstoy, E., Venn, K. A., Shetrone, M., et al. 2003, AJ, 125, 707

Tolstoy, E., Irwin, M. J., Helmi, A., et al. 2004, ApJ, 617, L119

Trager, S. C., King, I. R., \& Djorgovski, S. 1995, AJ, 109, 218

Valenti, J. A., Piskunov, N., \& Johns-Krull, C. M. 1998, ApJ, 498, 851

Venn, K. A., Irwin, M., Shetrone, M. D., et al. 2004, AJ, 128, 1177

White, S. D. M., \& Rees, M. J. 1978, MNRAS, 183, 341

Wiese, W. L., Smith, M. W., \& Miles, B. M. 1969, NSRDS-NBS 22 (NBS)

Wolnik, S. J., Berthel, R. O., \& Wares, G. W. 1971, ApJ, 166, L31 (WBW2)

Zaggia, S., Bonifacio, P., Bellazini, M., et al. 2004, Mem. Soc. Astron. It. Suppl., 5, 291

Zaritsky, D., \& Harris, J. 2004, ApJ, 604, 167 


\section{Online Material}


L. Monaco et al.: Bright RGB stars in the Sgr dSph, Online Material p 2

\section{Appendix A: Individual line data}

The following tables report the line list and adopted atomic parameters for the program stars. The measured equivalent width and the corresponding abundance obtained for each line are also reported. 
L. Monaco et al.: Bright RGB stars in the Sgr dSph, Online Material p 3

Table A.1. Line list and adopted atomic parameters for the program stars. The measured equivalent width and the corresponding abundance obtained for each line are also reported.

\begin{tabular}{|c|c|c|c|c|c|c|c|c|c|c|c|c|c|}
\hline Ion & $\begin{array}{r}\lambda \\
(\mathrm{nm})\end{array}$ & $\log g f$ & $\begin{array}{l}\text { Source of } \\
\log g f \\
\text { (see notes) }\end{array}$ & $\begin{array}{r}E W \\
(\mathrm{pm}) \\
2300127\end{array}$ & $\epsilon$ & $\begin{array}{r}E W \\
(\mathrm{pm}) \\
2300196\end{array}$ & $\epsilon$ & $\begin{array}{r}E W(\mathrm{pm}) \\
(\mathrm{pm}) \\
2300215\end{array}$ & $\epsilon$ & $\begin{array}{r}E W \\
(\mathrm{pm}) \\
2409744\end{array}$ & $\epsilon$ & $\begin{array}{r}E W \\
(\mathrm{pm}) \\
3600073^{\star}\end{array}$ & $\epsilon$ \\
\hline Fe I & 585.5076 & -1.76 & FMW & - & - & 5.89 & 7.375 & - & - & 4.42 & 7.253 & 4.44 & 7.149 \\
\hline $\mathrm{Fe} \mathrm{I}$ & 588.3817 & -1.36 & FMW & 9.19 & 6.607 & 10.70 & 6.763 & 11.67 & 7.195 & - & - & 10.66 & 6.861 \\
\hline $\mathrm{Fe} \mathrm{I}$ & 595.2718 & -1.44 & FMW & 6.51 & 6.269 & 9.42 & 6.684 & - & - & - & - & 7.29 & 6.398 \\
\hline $\mathrm{Fe} \mathrm{I}$ & 602.4058 & -0.12 & FMW & 11.52 & 6.654 & 15.93 & 7.153 & 13.97 & 7.173 & 14.75 & 7.367 & 11.14 & 6.569 \\
\hline $\mathrm{Fe} \mathrm{I}$ & 602.7051 & -1.21 & FMW & 12.20 & 7.15 & 14.34 & 7.335 & 11.75 & 7.214 & - & - & 9.30 & 6.637 \\
\hline $\mathrm{Fe} I$ & 605.6005 & -0.46 & FMW & 8.91 & 6.825 & 9.66 & 6.808 & 9.65 & 7.031 & - & - & 7.34 & 6.535 \\
\hline $\mathrm{Fe} I$ & 609.6664 & -1.93 & FMW & 5.98 & 6.666 & - & - & 9.26 & 7.352 & - & - & 6.18 & 6.702 \\
\hline $\mathrm{Fe} I$ & 615.1617 & -3.30 & FMW & 15.14 & 6.671 & 17.38 & 6.982 & 17.52 & 7.339 & 16.63 & 7.263 & 15.05 & 6.701 \\
\hline $\mathrm{Fe} I$ & 616.5360 & -1.55 & FMW & 7.46 & 6.772 & 9.78 & 7.066 & 9.20 & 7.19 & 8.86 & 7.172 & 6.80 & 6.657 \\
\hline $\mathrm{Fe} I$ & 618.7989 & -1.72 & FMW & 8.18 & 6.755 & 10.88 & 7.108 & 9.67 & 7.149 & 10.19 & 7.291 & 9.04 & 6.895 \\
\hline $\mathrm{Fe} \mathrm{I}$ & 622.6734 & -2.22 & FMW & 2.34 & 6.116 & 7.57 & 7.045 & 7.73 & 7.229 & 7.63 & 7.238 & 5.37 & 6.706 \\
\hline $\mathrm{Fe} \mathrm{I}$ & 651.8366 & -2.75 & FMW & 13.18 & 6.811 & 14.61 & 6.979 & 17.20 & 7.677 & 13.50 & 7.162 & 13.83 & 6.937 \\
\hline $\mathrm{Fe} I$ & 659.7559 & -1.07 & FMW & 5.97 & 7.017 & 6.07 & 6.968 & 7.34 & 7.32 & 6.72 & 7.248 & 4.54 & 6.747 \\
\hline $\mathrm{Fe} \mathrm{I}$ & 670.3566 & -3.16 & FMW & 11.49 & 6.823 & 13.50 & 7.107 & 14.97 & 7.632 & 12.19 & 7.225 & 11.57 & 6.856 \\
\hline $\mathrm{Fe} I$ & 673.9521 & -4.95 & FMW & 11.75 & 6.714 & 11.36 & 6.785 & - & - & - & - & 9.83 & 6.486 \\
\hline $\mathrm{Fe} \mathrm{I}$ & 674.6954 & -4.35 & FMW & - & - & 6.23 & 7.124 & - & - & 6.66 & 7.284 & 5.01 & 6.843 \\
\hline $\mathrm{Fe} \mathrm{I}$ & 679.3258 & -2.47 & FMW & 2.41 & 6.658 & - & - & 4.19 & 7.151 & - & - & 2.73 & 6.742 \\
\hline Mg I & 552.8405 & -0.522 & G03 & 23.92 & 6.67 & 25.91 & 6.811 & 24.36 & 6.827 & - & - & 20.64 & 6.485 \\
\hline Mg I & 571.1088 & -1.729 & G03 & 14.75 & 6.98 & 16.63 & 7.125 & 16.58 & 7.326 & 14.16 & 7.052 & 12.13 & 6.612 \\
\hline Mg I & 631.8717 & -1.945 & G03 & - & - & 7.78 & 7.279 & 5.24 & 7.012 & - & - & 4.74 & 6.868 \\
\hline $\mathrm{Mg} \mathrm{I}$ & 631.9237 & -2.165 & G03 & - & - & 3.67 & 6.913 & 3.73 & 6.981 & 4.49 & 7.12 & 2.68 & 6.709 \\
\hline $\mathrm{Ca} \mathrm{I}$ & 585.7451 & 0.240 & SR & 19.82 & 5.381 & 23.93 & 5.848 & 21.37 & 5.782 & 20.52 & 5.719 & 17.66 & 5.192 \\
\hline $\mathrm{Ca} \mathrm{I}$ & 586.7562 & -1.490 & G03 & 6.94 & 5.263 & 8.57 & 5.643 & 12.08 & 6.253 & 9.27 & 5.811 & 6.25 & 5.231 \\
\hline $\mathrm{Ca} \mathrm{I}$ & 612.2217 & -0.315 & SR & 30.67 & 5.327 & 30.52 & 5.576 & 41.66 & 6.086 & - & - & 26.93 & 5.298 \\
\hline $\mathrm{Ca} \mathrm{I}$ & 616.9042 & -0.797 & SR & 17.36 & 5.494 & 19.67 & 5.846 & 19.24 & 6.089 & 17.68 & 5.908 & 14.76 & 5.151 \\
\hline $\mathrm{Ca} \mathrm{I}$ & 643.9075 & 0.390 & SR & 26.02 & 5.338 & 29.40 & 5.736 & 28.96 & 5.854 & 28.92 & 5.861 & 24.25 & 5.322 \\
\hline $\mathrm{Ca} \mathrm{I}$ & 645.5558 & -1.290 & SR & 14.86 & 5.535 & 15.11 & 5.632 & 14.67 & 5.795 & 13.82 & 5.687 & 13.84 & 5.439 \\
\hline $\mathrm{Ca} \mathrm{I}$ & 649.3781 & -0.109 & SR & 21.22 & 5.289 & 21.69 & 5.351 & 22.97 & 5.812 & 22.43 & 5.795 & 18.13 & 4.933 \\
\hline $\mathrm{Ca} \mathrm{I}$ & 649.9650 & -0.818 & SR & 16.09 & 5.243 & 20.09 & 5.843 & 21.65 & 6.364 & 18.07 & 5.908 & 16.61 & 5.4 \\
\hline $\mathrm{Ca} \mathrm{I}$ & 650.8850 & -2.110 & NBS & 7.53 & 5.276 & 7.99 & 5.550 & 9.06 & 5.744 & 9.75 & 5.836 & 5.49 & 5.082 \\
\hline Ti I & 588.0269 & -2.045 & MFW & 13.16 & 4.002 & 14.99 & 4.512 & 16.08 & 4.985 & 14.31 & 4.684 & 9.68 & 3.591 \\
\hline $\mathrm{Ti} \mathrm{I}$ & 590.3315 & -2.145 & MFW & 12.61 & 4.029 & 16.09 & 4.789 & 12.98 & 4.544 & 13.38 & 4.624 & 10.91 & 3.884 \\
\hline $\mathrm{Ti} \mathrm{I}$ & 593.7809 & -1.890 & MFW & 13.52 & 3.903 & 15.31 & 4.403 & 15.86 & 4.761 & 15.38 & 4.705 & 13.95 & 4.088 \\
\hline Ti I & 595.3160 & -0.329 & MFW & 15.25 & 4.023 & 17.71 & 4.516 & 17.94 & 4.935 & 17.02 & 4.826 & 11.69 & 3.516 \\
\hline Ti I & 597.8541 & -0.496 & MFW & 14.21 & 3.981 & 15.55 & 4.325 & 17.77 & 5.039 & 15.03 & 4.567 & 12.59 & 3.8 \\
\hline $\mathrm{Ti} \mathrm{I}$ & 601.6995 & -3.630 & MFW & - & - & 3.21 & 4.615 & 4.47 & 4.783 & 3.78 & 4.611 & 1.69 & 3.956 \\
\hline Ti I & 606.4626 & -1.944 & MFW & - & - & 13.98 & 4.219 & 16.58 & 4.896 & 16.49 & 4.925 & 13.91 & 4.08 \\
\hline $\mathrm{Ti} \mathrm{I}$ & 609.1171 & -0.423 & MFW & 11.49 & 4.081 & 13.57 & 4.553 & 16.34 & 5.236 & 12.30 & 4.571 & 10.83 & 4.063 \\
\hline Ti I & 609.2792 & -1.379 & MFW & 7.80 & 3.89 & 10.40 & 4.511 & 8.53 & 4.35 & 9.65 & 4.487 & 7.01 & 3.89 \\
\hline
\end{tabular}


Table A.1. continued.

\begin{tabular}{|c|c|c|c|c|c|c|c|c|c|c|c|c|c|}
\hline Ion & $\begin{array}{r}\lambda \\
(\mathrm{nm})\end{array}$ & $\log g f$ & $\begin{array}{l}\text { Source of } \\
\log g f \\
\text { (see notes) }\end{array}$ & $\begin{array}{r}E W \\
(\mathrm{pm}) \\
3600230\end{array}$ & $\epsilon$ & $\begin{array}{r}E W \\
(\mathrm{pm}) \\
3600262\end{array}$ & $\epsilon$ & $\begin{array}{r}E W(\mathrm{pm}) \\
(\mathrm{pm}) \\
3600302\end{array}$ & $\epsilon$ & $\begin{array}{r}E W \\
(\mathrm{pm}) \\
3700178^{\star}\end{array}$ & $\epsilon$ & $\begin{array}{r}E W \\
(\mathrm{pm}) \\
00199\end{array}$ & $\epsilon$ \\
\hline Fe I & 585.5076 & -1.76 & FMW & 6.73 & 7.701 & - & - & - & - & 4.42 & 7.217 & 3.06 & 6.832 \\
\hline $\mathrm{Fe} \mathrm{I}$ & 588.3817 & -1.36 & FMW & 11.04 & 7.257 & 9.78 & 6.87 & 8.95 & 6.902 & 12.59 & 7.134 & 8.77 & 6.514 \\
\hline $\mathrm{Fe} \mathrm{I}$ & 595.2718 & -1.44 & FMW & 10.48 & 7.249 & 9.60 & 6.952 & 10.97 & 7.428 & - & - & 7.28 & 6.376 \\
\hline $\mathrm{Fe} I$ & 602.4058 & -0.12 & FMW & 14.35 & 7.397 & 14.91 & 7.335 & 11.72 & 7.037 & 12.74 & 6.777 & 9.52 & 6.18 \\
\hline $\mathrm{Fe} \mathrm{I}$ & 602.7051 & -1.21 & FMW & 10.62 & 7.166 & 10.34 & 6.98 & 9.77 & 7.077 & 11.18 & 6.929 & 7.11 & 6.235 \\
\hline $\mathrm{Fe} \mathrm{I}$ & 605.6005 & -0.46 & FMW & - & - & - & - & 9.99 & 7.307 & 9.79 & 6.939 & 5.76 & 6.147 \\
\hline $\mathrm{Fe} \mathrm{I}$ & 609.6664 & -1.93 & FMW & 9.35 & 7.493 & 7.49 & 7.071 & 8.41 & 7.388 & 8.95 & 7.174 & 4.23 & 6.378 \\
\hline $\mathrm{Fe} \mathrm{I}$ & 615.1617 & -3.30 & FMW & 15.16 & 7.221 & 15.05 & 6.991 & 14.02 & 7.062 & 18.70 & 7.176 & 11.90 & 6.428 \\
\hline Fe I & 616.5360 & -1.55 & FMW & 9.38 & 7.341 & 7.81 & 6.974 & - & - & 9.53 & 7.114 & 5.69 & 6.435 \\
\hline $\mathrm{Fe} \mathrm{I}$ & 618.7989 & -1.72 & FMW & 10.02 & 7.352 & 10.75 & 7.36 & 8.67 & 7.163 & 9.35 & 6.959 & 6.20 & 6.415 \\
\hline $\mathrm{Fe} \mathrm{I}$ & 622.6734 & -2.22 & FMW & 7.26 & 7.221 & 8.91 & 7.448 & 8.27 & 7.492 & 5.19 & 6.761 & 3.07 & 6.321 \\
\hline $\mathrm{Fe} \mathrm{I}$ & 651.8366 & -2.75 & FMW & 15.48 & 7.638 & 13.44 & 7.125 & 13.62 & 7.39 & 13.99 & 6.929 & 10.83 & 6.586 \\
\hline $\mathrm{Fe} \mathrm{I}$ & 659.7559 & -1.07 & FMW & 6.60 & 7.23 & 5.85 & 7.087 & 5.13 & 7.053 & 7.75 & 7.328 & 2.73 & 6.284 \\
\hline $\mathrm{Fe} \mathrm{I}$ & 670.3566 & -3.16 & FMW & 10.61 & 7.083 & 12.58 & 7.271 & 11.40 & 7.282 & 15.48 & 7.423 & 8.72 & 6.555 \\
\hline $\mathrm{Fe} \mathrm{I}$ & 673.9521 & -4.95 & FMW & 12.39 & 7.381 & 10.84 & 6.947 & 9.69 & 6.914 & 11.79 & 6.816 & 7.40 & 6.47 \\
\hline $\mathrm{Fe} \mathrm{I}$ & 674.6954 & -4.35 & FMW & 5.59 & 7.18 & 7.14 & 7.384 & 5.87 & 7.262 & 5.65 & 7.053 & - & - \\
\hline $\mathrm{Fe} I$ & 679.3258 & -2.47 & FMW & 6.63 & 7.608 & 4.56 & 7.238 & 4.00 & 7.196 & 4.92 & 7.243 & - & - \\
\hline Mg I & 552.8405 & -0.522 & G03 & 26.90 & 7.019 & - & - & 26.42 & 7.003 & 25.88 & 6.831 & 20.68 & 6.617 \\
\hline $\mathrm{Mg} \mathrm{I}$ & 571.1088 & -1.729 & G03 & 16.13 & 7.383 & 14.74 & 7.102 & 13.29 & 7.044 & 12.35 & 6.621 & 10.03 & 6.425 \\
\hline $\mathrm{Mg} \mathrm{I}$ & 631.8717 & -1.945 & G03 & 5.97 & 7.137 & 7.10 & 7.309 & 3.88 & 6.841 & 5.53 & 7.042 & 2.45 & 6.475 \\
\hline $\mathrm{Mg} \mathrm{I}$ & 631.9237 & -2.165 & G03 & 6.41 & 7.428 & 6.33 & 7.413 & 4.91 & 7.246 & - & - & 1.96 & 6.575 \\
\hline Ca I & 585.7451 & 0.240 & SR & 19.10 & 5.772 & 17.91 & 5.4 & 20.24 & 5.78 & 18.14 & 5.09 & 15.44 & 5.501 \\
\hline $\mathrm{Ca} \mathrm{I}$ & 586.7562 & -1.490 & G03 & 7.53 & 5.712 & 9.25 & 5.814 & 9.00 & 5.859 & 10.79 & 5.788 & 3.81 & 5.442 \\
\hline $\mathrm{Ca} \mathrm{I}$ & 612.2217 & -0.315 & SR & 30.57 & 5.856 & 26.93 & 5.432 & 31.74 & 5.737 & 41.80 & 5.91 & 24.84 & 5.805 \\
\hline $\mathrm{Ca} \mathrm{I}$ & 616.9042 & -0.797 & SR & 16.63 & 6.011 & 17.27 & 5.785 & 17.26 & 6.016 & 20.98 & 5.916 & 13.52 & 5.657 \\
\hline $\mathrm{Ca} \mathrm{I}$ & 643.9075 & 0.390 & SR & 24.07 & 5.723 & 23.66 & 5.415 & 28.40 & 5.894 & 27.02 & 5.399 & 21.83 & 5.641 \\
\hline $\mathrm{Ca} \mathrm{I}$ & 645.5558 & -1.290 & SR & 14.45 & 6.063 & 15.14 & 5.88 & 13.90 & 5.875 & 14.39 & 5.392 & 10.05 & 5.564 \\
\hline $\mathrm{Ca} \mathrm{I}$ & 649.3781 & -0.109 & SR & 20.98 & 5.653 & 19.90 & 5.418 & 20.23 & 5.679 & 20.86 & 5.125 & 18.14 & 5.604 \\
\hline $\mathrm{Ca} \mathrm{I}$ & 649.9650 & -0.818 & SR & 16.19 & 5.876 & 16.61 & 5.629 & 17.58 & 6.019 & 15.73 & 5.102 & 13.18 & 5.564 \\
\hline $\mathrm{Ca} \mathrm{I}$ & 650.8850 & -2.110 & NBS & 7.32 & 5.657 & 6.72 & 5.43 & 8.86 & 5.79 & 9.14 & 5.527 & - & - \\
\hline Ti I & 588.0269 & -2.045 & MFW & 12.47 & 4.671 & 11.71 & 4.235 & 11.48 & 4.349 & 13.54 & 4.106 & 8.06 & 4.387 \\
\hline Ti I & 590.3315 & -2.145 & MFW & 11.11 & 4.527 & 11.98 & 4.395 & 10.54 & 4.285 & 13.31 & 4.192 & 7.33 & 4.412 \\
\hline Ti I & 593.7809 & -1.890 & MFW & 13.78 & 4.754 & 12.89 & 4.274 & 14.18 & 4.709 & 14.58 & 4.102 & 8.04 & 4.24 \\
\hline Ti I & 595.3160 & -0.329 & MFW & 15.62 & 4.902 & 13.83 & 4.184 & 13.32 & 4.318 & 12.88 & 3.629 & 11.25 & 4.335 \\
\hline Ti I & 597.8541 & -0.496 & MFW & 13.19 & 4.531 & 12.35 & 4.068 & 14.25 & 4.649 & 15.84 & 4.209 & 8.98 & 4.144 \\
\hline Ti I & 601.6995 & -3.630 & MFW & 3.09 & 4.703 & 3.59 & 4.661 & 2.81 & 4.48 & 3.24 & 4.382 & - & - \\
\hline Ti I & 606.4626 & -1.944 & MFW & 13.25 & 4.655 & 14.44 & 4.532 & 13.73 & 4.628 & 14.89 & 4.147 & 10.04 & 4.502 \\
\hline Ti I & 609.1171 & -0.423 & MFW & 10.83 & 4.578 & 11.86 & 4.5 & 12.48 & 4.783 & 14.67 & 4.556 & 6.40 & 4.262 \\
\hline Ti I & 609.2792 & -1.379 & MFW & 7.39 & 4.374 & 7.72 & 4.253 & 9.62 & 4.606 & 8.87 & 4.141 & 3.56 & 4.275 \\
\hline
\end{tabular}


L. Monaco et al.: Bright RGB stars in the Sgr dSph, Online Material p 5

Table A.1. continued.

\begin{tabular}{|c|c|c|c|c|c|c|c|c|c|c|c|}
\hline Ion & $\begin{array}{r}\lambda \\
(\mathrm{nm})\end{array}$ & $\log g f$ & $\begin{array}{l}\text { Source of } \\
\log g f \\
\text { (see notes) }\end{array}$ & $\begin{array}{r}E W \\
(\mathrm{pm}) \\
3800204\end{array}$ & $\epsilon$ & $\begin{array}{r}E W \\
(\mathrm{pm}) \\
3800318\end{array}$ & $\epsilon$ & $\begin{array}{r}E W(\mathrm{pm}) \\
(\mathrm{pm}) \\
3800319\end{array}$ & $\epsilon$ & $\begin{array}{r}\begin{array}{r}E W \\
(\mathrm{pm})\end{array} \\
3800336^{\star}\end{array}$ & $\epsilon$ \\
\hline Fe I & 585.5076 & -1.76 & FMW & - & - & 4.61 & 7.241 & - & - & - & - \\
\hline $\mathrm{Fe} \mathrm{I}$ & 588.3817 & -1.36 & FMW & 7.89 & 6.138 & 9.56 & 6.771 & - & - & 8.71 & 6.641 \\
\hline $\mathrm{Fe} \mathrm{I}$ & 595.2718 & -1.44 & FMW & 5.21 & 5.89 & 8.47 & 6.692 & - & - & 8.23 & 6.675 \\
\hline $\mathrm{Fe} \mathrm{I}$ & 602.4058 & -0.12 & FMW & 9.69 & 5.95 & 14.80 & 7.274 & - & - & 12.01 & 6.835 \\
\hline $\mathrm{Fe} \mathrm{I}$ & 602.7051 & -1.21 & FMW & 5.84 & 5.867 & 9.99 & 6.858 & - & - & 10.99 & 7.049 \\
\hline $\mathrm{Fe} \mathrm{I}$ & 605.6005 & -0.46 & FMW & 5.00 & 5.896 & 9.71 & 7.008 & - & - & 7.55 & 6.689 \\
\hline $\mathrm{Fe} \mathrm{I}$ & 609.6664 & -1.93 & FMW & 12.91 & 6.007 & 7.39 & 6.993 & - & - & 7.17 & 6.986 \\
\hline $\mathrm{Fe} \mathrm{I}$ & 615.1617 & -3.30 & FMW & 1.13 & 5.933 & 15.52 & 6.987 & - & - & 16.32 & 7.04 \\
\hline $\mathrm{Fe} \mathrm{I}$ & 616.5360 & -1.55 & FMW & 4.00 & 6.027 & 7.74 & 6.9 & - & - & 7.25 & 6.853 \\
\hline $\mathrm{Fe} \mathrm{I}$ & 618.7989 & -1.72 & FMW & 4.86 & 6.05 & 9.24 & 7.033 & - & - & 7.53 & 6.771 \\
\hline $\mathrm{Fe} \mathrm{I}$ & 622.6734 & -2.22 & FMW & 2.25 & 6.014 & 7.37 & 7.128 & - & - & 7.11 & 7.112 \\
\hline $\mathrm{Fe} \mathrm{I}$ & 651.8366 & -2.75 & FMW & 9.51 & 6.086 & 12.81 & 6.947 & - & - & 12.48 & 6.862 \\
\hline $\mathrm{Fe} \mathrm{I}$ & 659.7559 & -1.07 & FMW & - & - & 5.63 & 6.994 & - & - & 5.52 & 7.041 \\
\hline $\mathrm{Fe} \mathrm{I}$ & 670.3566 & -3.16 & FMW & 6.42 & 6.005 & 11.91 & 7.083 & - & - & - & - \\
\hline $\mathrm{Fe} \mathrm{I}$ & 673.9521 & -4.95 & FMW & 5.76 & 5.998 & 9.47 & 6.648 & - & - & 10.34 & 6.71 \\
\hline $\mathrm{Fe} \mathrm{I}$ & 674.6954 & -4.35 & FMW & - & - & 5.72 & 7.096 & - & - & 5.85 & 7.103 \\
\hline $\mathrm{Fe} \mathrm{I}$ & 679.3258 & -2.47 & FMW & - & - & - & - & - & - & - & - \\
\hline $\mathrm{Mg} \mathrm{I}$ & 552.8405 & -0.522 & G03 & 19.95 & 6.239 & 25.04 & 6.858 & 18.45 & 6.469 & 24.82 & 6.866 \\
\hline $\mathrm{Mg} \mathrm{I}$ & 571.1088 & -1.729 & G03 & 10.90 & 6.313 & 14.70 & 7.06 & 10.81 & 6.653 & 14.66 & 7.101 \\
\hline $\mathrm{Mg} \mathrm{I}$ & 631.8717 & -1.945 & G03 & - & - & 3.54 & 6.702 & - & - & - & - \\
\hline $\mathrm{Mg} \mathrm{I}$ & 631.9237 & -2.165 & G03 & - & - & 2.38 & 6.681 & - & - & 4.07 & 7.067 \\
\hline $\mathrm{Ca} \mathrm{I}$ & 585.7451 & 0.240 & SR & 14.48 & 4.969 & 23.54 & 5.949 & 13.20 & 5.347 & 21.15 & 5.654 \\
\hline $\mathrm{Ca} \mathrm{I}$ & 586.7562 & -1.490 & G03 & - & - & 12.35 & 6.278 & - & - & 9.14 & 5.667 \\
\hline $\mathrm{Ca} \mathrm{I}$ & 612.2217 & -0.315 & SR & - & - & 29.75 & 5.621 & 18.71 & 5.267 & - & - \\
\hline $\mathrm{Ca} \mathrm{I}$ & 616.9042 & -0.797 & SR & 11.15 & 4.938 & 17.28 & 5.77 & 9.69 & 5.237 & 16.82 & 5.6 \\
\hline $\mathrm{Ca} \mathrm{I}$ & 643.9075 & 0.390 & SR & - & - & 28.72 & 5.848 & 17.55 & 5.28 & 25.90 & 5.529 \\
\hline $\mathrm{Ca} \mathrm{I}$ & 645.5558 & -1.290 & SR & 8.64 & 5.092 & 14.54 & 5.749 & 7.43 & 5.356 & 14.79 & 5.691 \\
\hline $\mathrm{Ca} \mathrm{I}$ & 649.3781 & -0.109 & SR & 16.22 & 4.873 & 23.18 & 5.826 & 15.61 & 5.477 & 22.53 & 5.658 \\
\hline $\mathrm{Ca} \mathrm{I}$ & 649.9650 & -0.818 & SR & 11.42 & 4.958 & 19.68 & 6.077 & 12.49 & 5.687 & 18.59 & 5.828 \\
\hline $\mathrm{Ca} \mathrm{I}$ & 650.8850 & -2.110 & NBS & - & - & 9.91 & 5.841 & - & - & 7.59 & 5.389 \\
\hline Ti I & 588.0269 & -2.045 & MFW & 3.97 & 3.56 & 15.47 & 4.822 & 3.66 & 4.069 & 14.00 & 4.405 \\
\hline Ti I & 590.3315 & -2.145 & MFW & 4.44 & 3.744 & 12.62 & 4.436 & 3.67 & 4.187 & 12.11 & 4.19 \\
\hline Ti I & 593.7809 & -1.890 & MFW & 4.94 & 3.55 & 15.34 & 4.621 & 4.30 & 4.029 & 14.55 & 4.336 \\
\hline Ti I & 595.3160 & -0.329 & MFW & 8.42 & 3.599 & 17.81 & 4.875 & 6.63 & 3.917 & 16.24 & 4.463 \\
\hline Ti I & 597.8541 & -0.496 & MFW & 6.43 & 3.515 & 16.98 & 4.849 & 7.17 & 4.14 & 14.47 & 4.261 \\
\hline Ti I & 601.6995 & -3.630 & MFW & - & - & 3.94 & 4.657 & - & - & 4.33 & 4.521 \\
\hline Ti I & 606.4626 & -1.944 & MFW & 6.83 & 3.78 & 16.48 & 4.829 & 6.06 & 4.293 & 16.14 & 4.627 \\
\hline Ti I & 609.1171 & -0.423 & MFW & 4.41 & 3.744 & 13.42 & 4.706 & 5.51 & 4.346 & 13.62 & 4.626 \\
\hline Ti I & 609.2792 & -1.379 & MFW & 1.94 & 3.711 & 9.20 & 4.403 & - & - & 8.93 & 4.214 \\
\hline
\end{tabular}


Table A.1. continued.

\begin{tabular}{|c|c|c|c|c|c|c|c|c|c|c|c|c|c|}
\hline Ion & $\begin{array}{r}\lambda \\
(\mathrm{nm})\end{array}$ & $\log g f$ & $\begin{array}{l}\text { Source of } \\
\log g f \\
\text { (see notes) }\end{array}$ & $\begin{array}{r}E W \\
(\mathrm{pm}) \\
4207953^{\star}\end{array}$ & $\epsilon$ & $\begin{array}{r}E W \\
(\mathrm{pm}) \\
4303773\end{array}$ & $\bar{\epsilon}$ & $\begin{array}{r}E W(\mathrm{pm}) \\
(\mathrm{pm}) \\
4304445\end{array}$ & $\epsilon$ & $\begin{array}{r}E W \\
(\mathrm{pm}) \\
4402285\end{array}$ & $\epsilon$ & $\begin{array}{r}E W \\
(\mathrm{pm}) \\
4408968\end{array}$ & $\epsilon$ \\
\hline Fe I & 585.5076 & -1.76 & FMW & 5.63 & 7.408 & 3.06 & 6.896 & 4.90 & 7.28 & 4.63 & 7.275 & 5.11 & 7.309 \\
\hline Fe I & 588.3817 & -1.36 & FMW & 11.23 & 7.057 & 10.20 & 6.836 & 11.99 & 7.326 & - & - & 12.23 & 7.2 \\
\hline $\mathrm{Fe} \mathrm{I}$ & 595.2718 & -1.44 & FMW & - & - & 8.60 & 6.666 & - & - & 9.46 & 7.076 & 8.97 & 6.75 \\
\hline $\mathrm{Fe} \mathrm{I}$ & 602.4058 & -0.12 & FMW & 12.90 & 6.953 & 13.07 & 6.936 & 13.48 & 7.139 & 13.24 & 7.238 & 14.22 & 7.087 \\
\hline $\mathrm{Fe} \mathrm{I}$ & 602.7051 & -1.21 & FMW & 11.81 & 7.169 & - & - & 11.50 & 7.219 & 10.76 & 7.235 & 10.57 & 6.91 \\
\hline $\mathrm{Fe} \mathrm{I}$ & 605.6005 & -0.46 & FMW & 8.70 & 6.822 & 8.52 & 6.741 & 9.16 & 6.936 & - & - & 10.45 & 7.048 \\
\hline $\mathrm{Fe} \mathrm{I}$ & 609.6664 & -1.93 & FMW & 7.32 & 6.959 & 6.15 & 6.739 & 8.17 & 7.164 & 7.72 & 7.191 & 8.38 & 7.137 \\
\hline $\mathrm{Fe} \mathrm{I}$ & 615.1617 & -3.30 & FMW & 16.99 & 7.166 & 14.48 & 6.781 & 13.99 & 6.905 & 13.75 & 7.143 & 16.13 & 7.114 \\
\hline $\mathrm{Fe} \mathrm{I}$ & 616.5360 & -1.55 & FMW & 8.92 & 7.082 & 5.83 & 6.531 & 8.76 & 7.113 & 9.79 & 7.447 & 9.19 & 7.105 \\
\hline $\mathrm{Fe} \mathrm{I}$ & 618.7989 & -1.72 & FMW & 9.47 & 7.052 & 7.88 & 6.751 & 10.19 & 7.27 & 9.90 & 7.366 & 9.18 & 6.993 \\
\hline Fe I & 622.6734 & -2.22 & FMW & 8.24 & 7.249 & 4.73 & 6.647 & 7.65 & 7.205 & 6.40 & 7.078 & 6.36 & 6.966 \\
\hline $\mathrm{Fe} \mathrm{I}$ & 651.8366 & -2.75 & FMW & 14.04 & 7.11 & 11.96 & 6.765 & 14.87 & 7.422 & 12.52 & 7.254 & 15.48 & 7.373 \\
\hline $\mathrm{Fe} \mathrm{I}$ & 659.7559 & -1.07 & FMW & 6.24 & 7.087 & - & - & 7.29 & 7.264 & 7.17 & 7.293 & 6.75 & 7.126 \\
\hline $\mathrm{Fe} \mathrm{I}$ & 670.3566 & -3.16 & FMW & 12.82 & 7.193 & 11.45 & 6.965 & 11.33 & 7.101 & 10.31 & 7.13 & 11.01 & 6.972 \\
\hline $\mathrm{Fe} \mathrm{I}$ & 673.9521 & -4.95 & FMW & - & - & 9.24 & 6.586 & 10.66 & 6.97 & 9.42 & 7.021 & 10.28 & 6.888 \\
\hline $\mathrm{Fe} \mathrm{I}$ & 674.6954 & -4.35 & FMW & 5.90 & 7.078 & - & - & 6.41 & 7.25 & 4.36 & 7.058 & 6.19 & 7.253 \\
\hline $\mathrm{Fe} \mathrm{I}$ & 679.3258 & -2.47 & FMW & 4.09 & 7.068 & 4.42 & 7.103 & 3.60 & 6.988 & 5.86 & 7.463 & 5.15 & 7.278 \\
\hline Mg I & 552.8405 & -0.522 & G03 & 26.88 & 6.953 & 21.07 & 6.563 & - & - & 24.18 & 6.949 & 23.99 & 6.79 \\
\hline $\mathrm{Mg} \mathrm{I}$ & 571.1088 & -1.729 & G03 & 13.37 & 6.858 & 13.48 & 6.856 & 15.72 & 7.268 & 13.61 & 7.108 & 12.82 & 6.774 \\
\hline $\mathrm{Mg} \mathrm{I}$ & 631.8717 & -1.945 & G03 & 6.26 & 7.135 & 2.48 & 6.456 & 6.43 & 7.161 & 7.58 & 7.389 & - & - \\
\hline $\mathrm{Mg} \mathrm{I}$ & 631.9237 & -2.165 & G03 & 3.64 & 6.927 & 2.94 & 6.775 & - & - & 3.80 & 6.995 & 3.20 & 6.862 \\
\hline $\mathrm{Ca} \mathrm{I}$ & 585.7451 & 0.240 & SR & 17.97 & 5.36 & 20.31 & 5.702 & 19.18 & 5.761 & 18.74 & 5.982 & 20.40 & 5.784 \\
\hline $\mathrm{Ca} \mathrm{I}$ & 586.7562 & -1.490 & G03 & 8.13 & 5.588 & 6.72 & 5.47 & 7.18 & 5.663 & 6.97 & 5.865 & 8.81 & 5.888 \\
\hline $\mathrm{Ca} \mathrm{I}$ & 612.2217 & -0.315 & SR & 34.59 & 5.836 & 27.12 & 5.529 & 28.52 & 5.794 & 24.80 & 5.827 & 30.80 & 5.847 \\
\hline $\mathrm{Ca} \mathrm{I}$ & 616.9042 & -0.797 & SR & 19.00 & 6.02 & 16.17 & 5.619 & 17.49 & 6.103 & 18.10 & 6.549 & 17.85 & 5.96 \\
\hline $\mathrm{Ca} \mathrm{I}$ & 643.9075 & 0.390 & SR & 26.92 & 5.7 & 23.20 & 5.406 & 23.37 & 5.637 & 24.34 & 6.013 & 25.33 & 5.678 \\
\hline $\mathrm{Ca} \mathrm{I}$ & 645.5558 & -1.290 & SR & 13.85 & 5.593 & 10.57 & 5.163 & 14.76 & 6.059 & 13.22 & 6.149 & 14.53 & 5.887 \\
\hline $\mathrm{Ca} \mathrm{I}$ & 649.3781 & -0.109 & SR & 20.17 & 5.405 & 19.33 & 5.347 & 20.40 & 5.755 & 17.64 & 5.704 & 21.24 & 5.675 \\
\hline $\mathrm{Ca} \mathrm{I}$ & 649.9650 & -0.818 & SR & 17.16 & 5.648 & 16.77 & 5.655 & 15.94 & 5.77 & 15.75 & 6.106 & 18.30 & 5.97 \\
\hline $\mathrm{Ca} \mathrm{I}$ & 650.8850 & -2.110 & NBS & 8.22 & 5.556 & 5.68 & 5.307 & 5.06 & 5.34 & 5.71 & 5.677 & 7.25 & 5.676 \\
\hline Ti I & 588.0269 & -2.045 & MFW & 16.14 & 4.879 & 10.28 & 4.003 & 11.33 & 4.419 & 9.77 & 4.594 & 11.74 & 4.461 \\
\hline $\mathrm{Ti} \mathrm{I}$ & 590.3315 & -2.145 & MFW & 13.87 & 4.574 & 8.84 & 3.92 & 10.86 & 4.457 & 10.49 & 4.837 & 12.52 & 4.692 \\
\hline $\mathrm{Ti} \mathrm{I}$ & 593.7809 & -1.890 & MFW & 14.79 & 4.453 & 10.84 & 3.938 & 11.13 & 4.231 & 11.23 & 4.7 & 13.69 & 4.597 \\
\hline Ti I & 595.3160 & -0.329 & MFW & 16.82 & 4.627 & 12.91 & 4.008 & 15.24 & 4.731 & 14.01 & 4.95 & 14.05 & 4.368 \\
\hline Ti I & 597.8541 & -0.496 & MFW & 14.40 & 4.309 & 11.65 & 3.942 & 13.06 & 4.444 & 12.22 & 4.722 & 13.10 & 4.357 \\
\hline $\mathrm{Ti} \mathrm{I}$ & 601.6995 & -3.630 & MFW & 3.52 & 4.51 & - & - & 1.70 & 4.429 & 1.85 & 4.818 & 1.95 & 4.63 \\
\hline Ti I & 606.4626 & -1.944 & MFW & - & - & - & - & 12.78 & 4.513 & 12.89 & 5.011 & 14.13 & 4.665 \\
\hline Ti I & 609.1171 & -0.423 & MFW & 12.29 & 4.462 & 8.95 & 4.05 & 10.64 & 4.515 & 10.41 & 4.853 & 11.87 & 4.662 \\
\hline Ti I & 609.2792 & -1.379 & MFW & 8.93 & 4.3 & 5.96 & 4.024 & 6.83 & 4.306 & 6.76 & 4.63 & 8.42 & 4.568 \\
\hline
\end{tabular}

* Star showing TiO molecular bands in the spectra.

FMW - Fuhr et al. (1988); G03 - Gratton et al. (2003); SR - Smith et al. (1981); NBS - Wiese et al. (1969); MFW - Martin et al. (1988). 
L. Monaco et al.: Bright RGB stars in the Sgr dSph, Online Material $p$

Table A.2. Iron line list and adopted atomic parameters for star \#3800319. The measured equivalent width and the corresponding abundance obtained for each line are also reported.

\begin{tabular}{|c|c|c|c|c|c|}
\hline Ion & $\begin{array}{r}\lambda \\
(\mathrm{nm})\end{array}$ & $\log g f$ & $\begin{array}{r}\text { Source of } \\
\log g f \\
\text { (see notes) }\end{array}$ & $\begin{array}{r}E W \\
(\mathrm{pm}) \\
3800319\end{array}$ & $\epsilon$ \\
\hline Fe I & 487.1318 & -0.410 & FMW & 17.32 & 5.949 \\
\hline $\mathrm{Fe} \mathrm{I}$ & 491.8994 & -0.370 & FMW & 17.84 & 5.958 \\
\hline $\mathrm{Fe} \mathrm{I}$ & 492.0502 & 0.060 & FMW & 30.56 & 6.368 \\
\hline $\mathrm{Fe} I$ & 495.7298 & -0.342 & WBW2 & 17.69 & 5.878 \\
\hline $\mathrm{Fe} \mathrm{I}$ & 495.7596 & 0.127 & WBW2 & 28.12 & 6.162 \\
\hline $\mathrm{Fe} \mathrm{I}$ & 500.6119 & -0.615 & FMW & 22.38 & 6.571 \\
\hline $\mathrm{Fe} \mathrm{I}$ & 501.2068 & -2.642 & FMW & 23.42 & 6.398 \\
\hline $\mathrm{Fe} I$ & 505.1635 & -2.795 & FMW & 22.54 & 6.528 \\
\hline $\mathrm{Fe} \mathrm{I}$ & 511.0413 & -3.760 & FMW & 18.78 & 5.715 \\
\hline $\mathrm{Fe} I$ & 517.1596 & -1.793 & FMW & 20.64 & 6.045 \\
\hline $\mathrm{Fe} I$ & 519.1454 & -0.551 & $\mathrm{O}$ & 18.57 & 6.362 \\
\hline $\mathrm{Fe} \mathrm{I}$ & 519.2344 & -0.421 & $\mathrm{O}$ & 14.89 & 5.643 \\
\hline $\mathrm{Fe} I$ & 519.4941 & -2.090 & FMW & 18.75 & 6.144 \\
\hline $\mathrm{Fe} \mathrm{I}$ & 522.7189 & -1.228 & $\mathrm{O}$ & 23.52 & 5.867 \\
\hline $\mathrm{Fe} \mathrm{I}$ & 523.2940 & -0.190 & FMW & 19.94 & 6.012 \\
\hline $\mathrm{Fe} I$ & 526.6555 & -0.490 & FMW & 19.96 & 6.379 \\
\hline $\mathrm{Fe} \mathrm{I}$ & 527.0356 & -1.510 & FMW & 25.96 & 6.378 \\
\hline $\mathrm{Fe} I$ & 532.4179 & -0.240 & FMW & 17.49 & 6.123 \\
\hline $\mathrm{Fe} I$ & 532.8039 & -1.466 & FMW & 43.04 & 6.118 \\
\hline $\mathrm{Fe} I$ & 532.8531 & -1.850 & $\mathrm{O}$ & 22.24 & 6.302 \\
\hline $\mathrm{Fe} \mathrm{I}$ & 537.1489 & -1.645 & FMW & 29.90 & 5.86 \\
\hline $\mathrm{Fe} I$ & 539.7128 & -1.993 & FMW & 26.15 & 5.884 \\
\hline $\mathrm{Fe} I$ & 540.5775 & -1.844 & FMW & 26.33 & 5.852 \\
\hline $\mathrm{Fe} \mathrm{I}$ & 542.9696 & -1.879 & FMW & 32.89 & 6.209 \\
\hline $\mathrm{Fe} I$ & 543.4524 & -2.122 & FMW & 22.59 & 5.806 \\
\hline $\mathrm{Fe} I$ & 544.6916 & -1.930 & FMW & 28.62 & 6.095 \\
\hline $\mathrm{Fe} \mathrm{I}$ & 545.5609 & -2.091 & $\mathrm{O}$ & 36.09 & 6.604 \\
\hline $\mathrm{Fe} I$ & 561.5644 & -0.140 & FMW & 19.44 & 6.333 \\
\hline $\mathrm{Fe} I$ & 595.2718 & -1.440 & FMW & 5.11 & 6.135 \\
\hline $\mathrm{Fe} I$ & 602.7051 & -1.210 & FMW & 6.22 & 6.195 \\
\hline $\mathrm{Fe} I$ & 616.5360 & -1.550 & FMW & 5.95 & 6.57 \\
\hline $\mathrm{Fe} \mathrm{I}$ & 670.3566 & -3.160 & FMW & 4.69 & 6.127 \\
\hline
\end{tabular}

FMW - Fuhr et al. (1988); WBW2 - Wolniket et al. (1971); O - O’Brian et al. (1991). 